Article

\title{
Ferromagnetic hysteresis by Heisenberg partition function and its processing methods in nanoparticle magnetization modeling
}

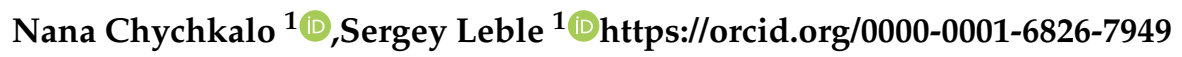 \\ ${ }^{1}$ Institute of Physics, Mathematics and IT Technology, Immanuel Kant Baltic Federal University, 236041 \\ Kaliningrad, Russian Federation \\ * Correspondence: lebleu@mail.ru \\ $\ddagger$ These authors contributed equally to this work.
}

\begin{abstract}
The Heisenberg ab initio theory of magnetization is developed to apply for multilayer nanoparticles. The theory is based on distribution and partition functions modification with account the difference between exchange integral and closest neighbour numbers, that change the system of resulting transcendental equation for magnetization and its reversal to form either a paramagnetic type curve or hysteresis loops patterns. The equations are obtained within the Heisenberg partition function construction by Heitler diagonalization of energy matrix via irreducible representations of permutation symmetry group. A combination with the Gauss distribution gives the explicit expression for the partition function in the asymptotic limit at large spin range in terms of transcendent function. The exchange integral, as a parameter of the equation of state (material equation) is evaluated from Curie temperature value by means of a formula derived within the presented theory. Methods of data processing from the simultaneous solution of the material equation system are proposed. The multi-valued function of hysteresis loop is found by combination of graphical approach and special procedure for elimination of mistaken peaks and prolapses of the patterns. The theory and computation methods are applied to spherical particles with separate surface layers consideration. The contribution of the surface layers, that are specified by number of closest neighbors and exchange integrals into overall magnetization, is studied for two-layer and three-layer models, that are discussed and compared graphically.
\end{abstract}

Keywords: hysteresis; Heisenberg magnetization theory; ab initio theory; multilayer models of nanoparticle; Curie-Weiss transition range;

\section{Introduction}

The phenomenon of hysteresis is important in many fields of physical science from ferromagnetism [1] (some advances are pointed out in [2]) to acoustics [3,4] and plasma waves theory [5]. The principle feature of the phenomenon is that the material relation in such cases is nonlinear and not unique, hence its visualization and applications need special attention. It is important to note the impact of these features on magnetization dynamics [1].

In the comprehensive and very instructive book on hysteresis in ferromagnets [1] it is pointed, that "the fascinating richness of phenomena' attract attention of many investigators as its fundamental physics side as in applications. In this book the problem of a hysteresis loop definition and, hence, drawing is posed.

In the book [6] few popular models, from Stoner - Wohlfarth' (especially important for nanoparticles) [7] are described in the practical context with a phenomenology including and 
comparison with experiments. In the case of particles of dimensions small enough to have the only domain, the theory significantly simplifies, hence the very many models are applied, starting from the mentioned [7]. How it works in a wide field of physics, it is shown in the review [8] and its features for ferromagnetic bodies are specified at [9].

Difficulties in a partition function ab initio construction are well-known [10]. The seminal paper of Heisenberg [11] on ferromagnetic matter theory is important as from widely explored and cited exchange interaction of the Weiss molecular forces interpretation as from less known implementation of permutation group symmetry in the partition function composition. It was established that the Weiss electric forces originated from exchange effect of quantum mechanics, introduced for the first time by Heitler and London [12]. The paper of Heisenberg [11] contains deep results of general significance. The multi-electron terms theory was built using the common symmetry: in respect to the group of permutation of the electrons. Its development is studied in [13] for more general exposition, to be valid for crystals. It has an extension based on a joint symmetry group of permutations and a space symmetry group [14], where the exchange integral notion is "lifted" up the Hartree-Fock equations level. The permutation group theory allows to express energy via its irreducible representations characters $[15,16]$ and, in same context, statistical distribution function is constructed. Its derivative gives the internal parameter (magnetisation $M$ value conjugate to magnetic field $H$, that results in equation of state $M(H)$. Its development for particles with unit spin was realized in [17]. In such approach, the basic element of the partition sum construction is the symmetry with respect to electron permutation group. However, the calculation contains account of relative positions and number of neighbours, that enter the resulting formulas, i.e. the equations that define the form of a magnetisation curve or a hysteresis loop. In a sense such a construction accounts anisotropy in implicit form, that, in the simplest models as Stoner one, led directly to the hysteresis phenomenon for a one-domain particles magnetization. A further development of the Heisenberg theory implies explicit account of a space symmetry [14], that would allow to introduce anisotropy parameters within the partition function. Some basic observations and modeling outline for nanoscale objects are presented in [14,18]. Note, that at huge values of the spin, the problem may be considered as the quasiclassical approximation as it does in the transition to the continuous description (e.g. while a derivation of LLG [19] is performed). The microscopic Heisenberg model goes to the macroscopic Landau-Lifshits-Gilbert (LLG) equations as it is explained in $[19,20]$. For example, in the article [21] a ferromagnetic alloy explore such LLG-based model for a hysteresis loop building with 3D LLG equations. Further, magnetic bi-phase nanocrystalline systems with biased (non-symmetric) hysteresis loops are presented in [22], where micromagnetic computations of magnetostatic and exchange interactions have been made within the model using Euler-Lagrange equations for classic Lagrangean.

Typically, a nano- or microwire have a magnetic structure consisting mainly of a single longitudinal domain and small closure domains at both ends. A rectangular hysteresis loop is then observed accompanied by the fast domain wall propagation along the wires. This type of material is a good physical example of the idea of a magnetic relay hysteron as described in Preisach model [23] hysteresis. As example of a core/shell system that shows magnetization reversal (strongly affected by the presence of the shell) and, in particular, by the existence of a frustrated interfacial region playing a key role in determining the low temperature irreversibility, the finite coercitivity slightly above the Curie temperature of the phase and the horizontal displacement of the FC-hysteresis loop, which is attributed to the presence of a large fraction of surface spins [24]. In [25] The magnetization reversal sequence of the two ferromagnetic layers depends on the composition of a Permalloy. The magnetic features are explained in terms of morphological differences of the ferromagnet/antiferromagnet interfaces.

The main aim of our investigation is to attract attention to the "ab initio" modeling of a variety of hysteresis problems formulation and loops construction in a field of its direct application possibility. This field covers the one-domain case of magnetization and reversal, especially for nanoparticles which surface layer may contribute in resulting loops features. A necessity of such investigation is 
demonstrated experimentally, see, e.g., the mentioned paper [24]. The model we present in this study is based on a division of internal and surface layers of atoms with different exchange and neighbours number characteristics, that enter Heisenberg theory parameters. This model we estimate as novel, considering it as the main result of the presented work. We also develop results of Heisenberg and his followers in the realm of the hysteresis loops patterns construction for different ferromagnets and parameters range, including the vicinity of the ferromagnetic and paramagnetic border. Such results are also absent in literature, hence - new. It also was instructive to explain our choice of the corresponding thermodynamic relations for magnetization and strength of magnetic fields. The term "ab initio" is conventionally used in the literature in two meanings: 1 . Within a quantum mechanics scope, such as based on either Hartree - Fock approach, or in its development as DFT - pseudopotentials calculations [26], 2. By the foundation of classical/quantum statistics as in our work. The choice of equation and a statement of problem for a distribution function fix the realm of theory validity, such as kinetic or equilibrium Gibbs one (our case).

In this manuscript we also study the computational aspect of the hysteresis phenomenon visualisation, However, for a reader convenience, we "order" the basic physical footings of an "ab initio" theory, e.g. the partition function form origin, introducing the necessary notations and the choice of the corresponding thermodynamic relation for magnetization and strength fields to have the $M(H)$ equation of state. In the Second section we, for the same reason sketch the principle points of the Heisenberg ferromagnetism theory. This model contains hysteresis specific constructive elements, that have not been presented in original publications of Heisenberg and his followers, The Third section is devoted to direct corollaries of the theory basic relations in Curie-Weiss range, that allows express the exchange integral via the Curie temperature, study the law and illustrate the magnetization behavior in the vicinity of the critical temperature. We also pose and solve the problem of graphical solution of Heisenberg equations outside the ferromagnetism temperature region. In the Fourth, central point section, the general mathematical problems of magnetization visualization in the ferromagnetism region are presented. As the fourth one, the fifth section describes magnetization of nanoparticles in multi-layers model. A contribution of a surface in magnetism is of significant interest for the "tiny" particles. The layers introduced to figure out the difference between parameters of the atomic pairs at internal and external layers. Going to the sixth section, we stress its computational aspect, analysing such artefacts of the multi-valued nonlinear problems visualisation as prolapses and mistaken peaks generation by computing workflow. Such a tool for the problem overcome we name the subtraction and summation checking procedure, that we consider as also new. The Conclusion shortly review the results and outline perspectives. The short description of the overall algorithm is available online in [27]. The more complicated problem of Nonlinear Magnetization Dynamics in Nanosystems is presented in comprehensive book of Bertotti-Serpico [28]. We, however do not touch such problems with time-dependence in this text.

\section{Mathematical and physical background. Energy partitioning through the distribution function}

\subsection{Thermodynamic state equation}

A partition function $\mathrm{Z}$ is represented in quantum statistical physics as the normalisation constant of the distribution:

$$
\hat{g}(a, T)=\frac{1}{Z} \exp \left[-\frac{\hat{\mathcal{H}}(a)}{k T}\right]
$$

where $\hat{g}$ is a Gibbs canonical distribution operator, used for description of equilibrium states of physical systems. In (1), we note $\hat{\mathcal{H}}$ as the Hamiltonian operator, $k$ - Boltzmann constant, $T$ - absolute temperature. The Gibbs operator depends on external parameter $a$ of the system under consideration. 
We left the only such parameter in the magnetism phenomena description, its choice is done below. The normalization condition for (1) reads:

$$
\operatorname{Tr}[\hat{g}(a, T)]=\frac{1}{Z} \operatorname{Tr}\left[\exp \left[-\frac{\hat{\mathcal{H}}(V, a)}{k T}\right]\right]=1 .
$$

The thermodynamic internal energy of a system $U$ in the statistical approach is represented as the mean value of Hamiltonian:

$$
U=<\hat{\mathcal{H}}>=\operatorname{Tr}\left[\hat{\mathcal{H}} \cdot \exp \left[-\frac{F-\hat{\mathcal{H}}}{k T}\right]\right],
$$

where $F=U-T S$ ( $S$ - entropy), $F$ is a thermodynamic potential, Helmholtz free energy. Being the function of state, its differential is exact. Other thermodynamic variables are defined similarly to (3), as the mean values of corresponding operators. Proceeding the previous point, the connection between the free energy and the partition function is settled as: $F=k T \ln \frac{1}{Z}$. Plugging it in (2) yields

$$
\exp \left[\frac{F}{k T}\right] \operatorname{Tr}\left[\exp \left[-\frac{\hat{\mathcal{H}}}{k T}\right]\right]=1 .
$$

The identity (4) is differentiated, taking the tiny change of temperature $d T$ and external parameter $\delta a$ :

$$
d \frac{F}{k T}-\frac{1}{k T}<\frac{\partial \hat{\mathcal{H}}}{\partial a}>\delta a-<\hat{\mathcal{H}}>d \frac{1}{k T}=0 .
$$

The work $\delta A$ is defined by

$$
\delta A=<\frac{\partial \hat{\mathcal{H}}}{\partial a}>\delta a=-b \delta a,
$$

where $b$ is the internal parameter. As the result, the first law of thermodynamics is obtained:

$$
d U-T d S=\delta A .
$$

Then, as the Pfaff's form of free energy F is exact, it could be written as follows:

$$
d \frac{F(T, a)}{k T}=\frac{1}{k T} \frac{\partial F}{\partial a} d a+\frac{\partial F}{\partial T} d T+F d \frac{1}{k T}
$$

The following expression is substituted in the derived first law of thermodynamics (7), accounting the rule for linear combinations of independent differentials $d a$ and $d T$, by which the pairs of variables can be further correspondingly equated as:

$$
\left(\frac{\partial F}{\partial a}+b\right) d a+\left(\frac{\partial F}{\partial T}+S\right) d T=0
$$

On this ground, the state equation is derived:

$$
b=k T \frac{\partial \ln Z}{\partial a} .
$$

The pair of parameters, necessary for current magnetization patterns visualization, are $M=b, H=a$. $M$ - is the magnetization, $H$ - is the external magnetic field force,

$$
M=k T \frac{\partial \ln Z}{\partial H} .
$$

As the result, the connection between prominent quantities - the magnetization and the external magnetic field force - is set. 
Table 1. Number of closest neighbors $z$ by Heitler-London estimations

\begin{tabular}{|l|l|}
\hline$z$ & Application case $^{\#}$ \\
\hline 1 & Two atoms \\
\hline 2 & Linear chain \\
\hline 4 & Quadratic surface lattice \\
\hline 6 & Simple cubic lattice \\
\hline 8 & Cubic space-centered lattice \\
\hline 12 & Cubic face-centered lattice \\
\hline \#Correspondent condensed matter structure for listed $z$ values substitution.
\end{tabular}

\subsection{Heisenberg partition function and exchange interactions properties}

W. Heisenberg builds foundations of the ferromagnetic theory, basing on the general quantum statistics fundamentals, described in the preceded section with the effective use of universal symmetry of a multi-electron system with respect to the permutation group and the Pauli principle, which makes it generally applicable for various materials [11,29]. He incorporates the Heitler-London [12] results for exchange interaction description and, as the main tool for energy spectrum evaluation, the Heitler's one [15], that express the multi-electron Hamiltonian in terms of exchange integrals and characters of irreducible representations of the permutation group.

The exchange interaction is described by the number of closest neighbors $z$ - it is settled at the table 1. Note also, that the exchange integral $J$ - decreases exponentially with the increasing distance between atoms. The number of closest neighbors for Iron is $z_{F e}=8$, for Nickel and Cobalt $z_{N i}=z_{C o}=12$, basing on the knowledge about particular materials crystal structure; it is visualized on Figures 23 (Iron) and 24 (Nickel and Cobalt), placed in Appendix.

Heisenberg derives an expression for energy with the help of the characters of irreducible representations, listed in [15] and corrected in [11]. Its distribution within the $\sigma$-subspace is given by Gauss function. The partition function $Z$ by such construction is:

$$
Z=\sum_{s=0}^{n} \sum_{m=-s}^{+s} \int_{-\infty}^{+\infty} d \Delta E \frac{f_{\sigma}}{\sqrt{2 \pi \overline{\Delta E_{\sigma}^{2}}}} e^{\alpha m+\beta \frac{s^{2}}{2 \pi}-\frac{\Delta E}{k T}-\frac{\Delta E^{2}}{2 \Delta E_{\sigma}^{2}}}=\sum_{s=0}^{n} \sum_{m=-s}^{+s} f_{\sigma} e^{\alpha m+\beta \frac{s^{2}}{2 \pi}-\frac{\overline{\Delta E_{\sigma}^{2}}}{2 k^{2} T^{2}}}
$$

$m$ - magnetic quantum number, which denotes spin projections, $\overline{\Delta E^{2}}$ - mean quadratic energy deviation, $f_{\sigma}$ - dimension of the irreducible representation, $s$ - maximum value of $m, n$ - number of electrons, $k$ - the Boltzmann constant, $T$ - Kelvin temperature. Two assignations of dimensionless variables are made:

$$
\beta=\frac{z J}{k T}
$$

and

$$
\alpha=\frac{\mu_{B}}{k T} H
$$

here $\beta$ is a combination of internal material parameters, $\alpha$ - the dimensionless external magnetic field $(H), \mu_{B}$ - the Bohr magneton. After the summation of (12), we arrive at

$$
Z=K\left[2 \cosh \frac{\alpha+\beta \frac{m_{0}}{n}-\beta^{2} \frac{m_{0}}{n z}+\beta^{2} \frac{m_{0}^{3}}{2 n^{3} z}}{2}\right]^{2 n} .
$$

The most probable magnetic quantum number $m_{0}$ is determined by

$$
m_{0}=\frac{\partial \ln Z}{\partial \alpha}=\frac{\partial \ln \left([2 \cosh \xi]^{2 n}\right)}{\partial \alpha}=n \tanh \xi
$$



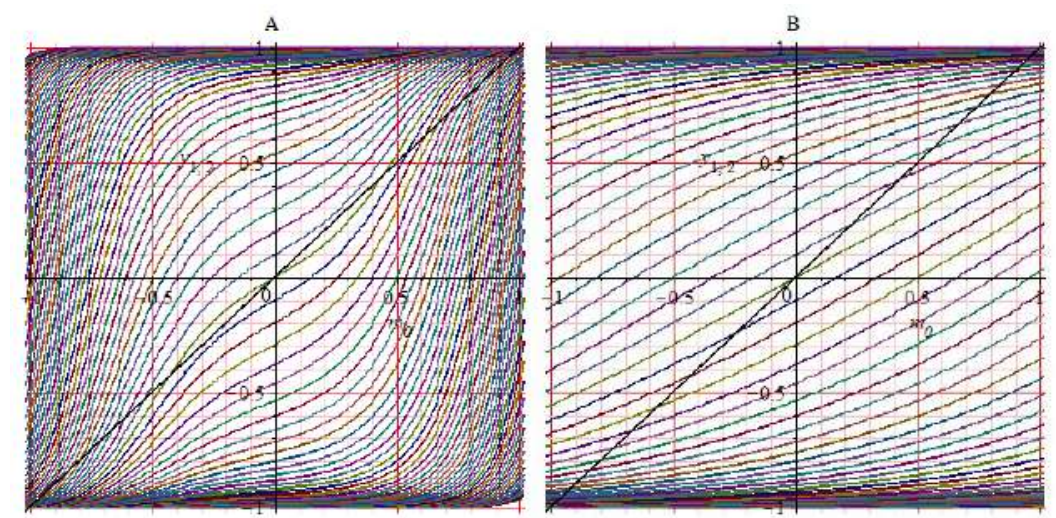

Figure 1. The function $\tanh \xi$ smoothness for an arbitrary material. A) $\beta=10.6, \mathrm{~B}) \beta=1.32$. With the decrease of $\beta, \tanh \xi$ becomes straitened and intersects the black line once. The thickening regions of $\tanh \xi$ functions correspond to the saturation of $m_{0}$, as they intersect the black line at the same value of $m_{0}$.

that is a form of the equation of state $m_{0}(\alpha)$ in terms of the dimensionless pair of quantities. Next, note, that the symbol $K$ is a function, which logarithm is small comparatively with the second term at large $n$ and $m_{0} / n$. It does not depend on $m_{0}$, so it is omitted. As in computations we mostly worked with $Z$, one more notation is introduced for the argument of hyperbolic function:

$$
\xi=\frac{\alpha+\beta \frac{m_{0}}{n}-\beta^{2} \frac{m_{0}}{n z}\left(1+\frac{m_{0}^{2}}{2 n^{2}}\right)}{2}
$$

\section{Para-Ferromagnetism border}

\subsection{Exchange interactions by Curie point characteristics}

The distinctive feature of Heisenberg ferromagnetic theory is in possibility of switching between paramagnetic and ferromagnetic magnetization states that defines the values of $\beta$ at the boundaries between paramagnetic and ferromagnetic ranges.

The terms $\beta \frac{m_{0}}{n}$ and $\beta^{2} \frac{m_{0}}{n z}$ define the smoothness of hyperbolic tangent functions with argument $\xi$. In turn, the smoothness of hyperbolic tangent functions determines whether $m_{0}$ is single-valued at each solution or if exists a set of $m_{0}$ values - Figure 1.

To define the parameter $\beta$ values at borders of switching from a paramagnetic state into a ferromagnetic one, we work with the condition:

$$
\beta\left(1-\frac{\beta}{z}\right) \geq 2
$$

As it is seen from equations $(16,17)$, the tangent of the curve (17) for $\frac{m_{0}}{n}=0$ goes under a smaller angle with the x-axis than the tangent of the curve (16), that define number of intersections of the curves, hence - the magnetization kind. Below we give a lot of examples of the case. The condition (18) is equated in the critical temperature point $T=\theta$ and $\beta$ here gets the assignation $\beta_{\theta}$. It brings us to the quadratic equation:

$$
\beta_{\theta}^{2}-z \beta_{\theta}+2 z=0 .
$$


Table 2. Exchange integrals $J$ through critical temperature $\theta$, see*

\begin{tabular}{|l|l|l|l|}
\hline & Critical point $\theta$ & Melting point & Exchange integral $J$ \\
\hline Units (in SI) & {$[$ Kelvin] } & {$[$ Kelvin] } & {$[$ Joule $]$} \\
\hline Iron & 1043 & 1538 & $7.19 \cdot 10^{-21}$ \\
\hline Nickel & 627 & 1455 & $1.82 \cdot 10^{-21}$ \\
\hline Cobalt & 1121 or $1403^{*}$ & 1495 & $3.26 \cdot 10^{-21}$ \\
\hline
\end{tabular}

The previously mentioned expression with square root $\sqrt{1-\frac{8}{z}}$ figures further in computations by the Heisenberg theory (inclusively for the exchange integral $J$ evaluation) as the consequence of a quadratic equation solution way via the discriminant. The solution consists of a pair of roots:

$$
\beta_{\theta}^{[1,2]}=\frac{z}{2}\left(1 \pm \sqrt{1-\frac{8}{z}}\right) .
$$

General expression $\beta=\frac{z J}{k T}$ in critical point takes the form:

$$
\beta_{\theta}^{[1,2]}=\frac{z J}{k \theta^{[1,2]}},
$$

and, with the roots (20) application for the exchange integral values we get:

$$
J=\frac{k \theta^{[1,2]}}{2}\left(1 \pm \sqrt{1-\frac{8}{z}}\right) .
$$

We, as W. Heisenberg, take one of two possible roots as physical one. Then, we work with the critical point value. An approximate estimation of exchange integral $J$ was given in [11] on base of general thermodynamics, it is indicated that two conditions have to be accounted for ferromagnetism existence. The first one is $J \sim k T$, where $k T$ assumes values of the order $10^{-13}[\mathrm{erg}]$ (in Gaussian system of units) degrees for Iron, Nickel and Cobalt materials. It follows that $J \sim 10^{-20}$ [Joule] (in System International).

Though, the expression (22) allows us to compute the concrete exchange integral $J$ value, which is further used in mean magnetization per electron and for a bulk layers in a multi-shell particle model as well - Table 2. Theoretically it is more suitable to express the approximate $J$ through the exact empirically measured $\theta$ point, established firstly for the materials by Pierre Curie [30].

1

For defining the most convenient values of $\beta_{\theta}$, we plug the numbers of closest neighbors $z$ values in (20), which are: If $z=8$, then

$$
\beta_{\theta}=4 \pm \sqrt{16-16}=4
$$

If $z=10$, then

$$
\beta_{\theta}^{[1,2]}=5 \pm \sqrt{25-20}=5 \pm \sqrt{5}
$$

If $z=12$, then

$$
\beta_{\theta}^{[1,2]}=6 \pm \sqrt{36-24}=6 \pm 2 \sqrt{3} .
$$

According to Table 1: when $z=8$ (corresponds to Iron material), $\beta_{\theta}$ has one root; when $z=12$ (corresponds to Nickel or Cobalt materials), $\beta_{\theta}^{[1,2]}$ has two roots.

$1 *$ The values from Table 2 might differ in various references. For our aim (the estimation of a model) implied values have no principle significance. 


\subsection{Heisenberg model with and without Curie-Weiss approximation}

In a primary source the Taylor expansion is done for the expression (16), taking small values of $\xi$. It brings us to the approximate description of a high temperature region by Heisenberg theory (namely, higher than Curie point temperatures):

$$
2 x=\alpha+\left(x-\frac{x^{3}}{3}\right)\left(\beta_{\theta}-\frac{\beta_{\theta}^{2}}{z}\right)+\left(x-\frac{x^{3}}{3}\right)^{3} \frac{\beta_{\theta}^{2}}{2 z} .
$$

The cubic and higher terms are discarded due to its minor significance, i.e.:

$$
m_{0} \approx x=\frac{\alpha}{2-\left(\beta_{\theta}-\frac{\beta_{\theta}^{2}}{z}\right)} .
$$

Further the $m_{0}$ is equated with the expression for $x$. Referring to the previous point of exchange integral $J$ reevaluation, $\beta_{\theta}$ is expressed here through

$$
\beta_{\theta}=\frac{z \theta}{2 T}\left(1 \pm \sqrt{1-\frac{8}{z}}\right)
$$

Then, by the binomial factoring rule, substitution of $\beta_{\theta}$ from (28), putting $\frac{1}{T}$ out of the brackets, tear minus out of the brackets to exchange the positions of $T$ and $\theta$ in $\theta-T$, the resultant expression of approximation is

$$
m_{0}=\frac{4 \alpha T^{2}}{z(T-\theta)\left(1-\sqrt{1-\frac{8}{z}}\right)\left(T\left(1+\sqrt{1-\frac{8}{z}}\right)-\theta\left(1-\sqrt{1-\frac{8}{z}}\right)\right)}
$$

For Iron $(z=8)$ it contracts to

$$
m_{0}=\frac{\alpha T^{2}}{2(T-\theta)^{2}}
$$

On the other hand, the $\xi$ expression (17) is to be prepared for high temperature magnetization without any approximations. Similarly, we substitute $\beta_{\theta}$ from (28):

$$
\xi_{\theta}=\frac{\alpha+\frac{z \theta}{2 T}\left(1 \pm \sqrt{1-\frac{8}{z}}\right) \frac{m_{0}}{n}-\left(\frac{z \theta}{2 T}\left(1 \pm \sqrt{1-\frac{8}{z}}\right)\right)^{2} \frac{m_{0}}{n z}+\left(\frac{z \theta}{2 T}\left(1 \pm \sqrt{1-\frac{8}{z}}\right)\right)^{2} \frac{m_{0}^{3}}{2 n^{3} z}}{2}
$$

and include the last expression in the system of two equations (the visualization of computation results is given in Figure 26 and Figure 27) of Appendix:

$$
y_{1}=\frac{m_{0}}{n} ; y_{2}=\tanh \xi_{\theta}
$$

The system is solved with the temperature change, accounting that $\alpha$ parameter should be small; for example, $\alpha=0.01$. The mean magnetization value is taken per one electron, so $n=1$, as we compute the statistical mean per one electron (see the next section for details). The result is obtained from the array of intersection points of the system (32), which is illustrated as the resulting figure of this section - Figure 2.

So, (32) and (29) are graphically compared, showing the difference in a curvature angle. The Curie-Weiss approximation gives a more uniform decrease of a function, and both graphs match with tending to the melting point of a material - Figure 2. 


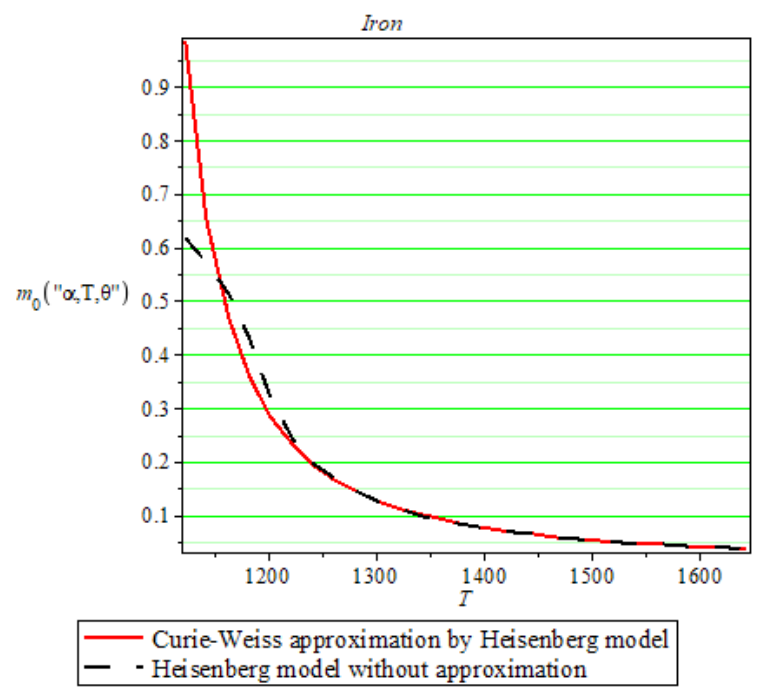

Figure 2. Visual comparison of $m_{0}(\theta, T, \alpha)$ dependence by Heisenberg model with application of Curie-Weiss approximation and without approximation. $T=1123 . .1643[K], \alpha=0.01, n=1$. Both functions almost coincide within the range $T>1250[K]$, by tending to zero, when the material melts.

\section{Mean per electron magnetization}

\subsection{Mean partition function per electron and functions set mathematical behavior}

For magnetization patterns estimations we consider that for one atom there is one active electron, which is involved in paramagnetism and ferromagnetism, as the other electrons magnetic properties are considered balanced with each other, and the resultant magnetic moment of an atom is equated to para- or ferromagnetism of one active electron. That is why we regard the mean magnetization per atom with $n=1$, where $n$-a number of active para-ferromagnetic electrons.

The expression (17) for mean magnetization per electron acquire the next method application: substituting the partition function with $n=1\left(Z_{1}\right)$ :

$$
\xi_{1}=\frac{\alpha+\beta m_{0}-\beta^{2} \frac{m_{0}}{z}+\beta^{2} \frac{m_{0}^{3}}{2 z}}{2}
$$

The most probable magnetic quantum number $m_{0}$ per one electron is determined by (16) is

$$
m_{0}=\frac{\partial \ln Z_{1}}{\partial \alpha}=\frac{\partial \ln \left(\left[2 \cosh \xi_{1}\right]^{2}\right)}{\partial \alpha}=\frac{2\left(2 \cosh \xi_{1}\right)^{2-1} \cdot 2 \sinh \xi_{1}}{2\left(\cosh \xi_{1}\right)^{2}}=\tanh \xi_{1}
$$

as it is a derivative of a compound function. Next, we compose the system of two equations:

$$
y_{1}=\tanh \xi_{1} ; y_{2}=m_{0}
$$

where $m_{0}$ is the main variable. The system (35) is considered to be the most convenient for software to find out the solutions in case of paramagnetic curves creation, but for the ferromagnetic case we recommend to imply the second way of gaining the intersection points with the usage of the system with inverted hyperbolic function:

$$
y_{1}=\xi_{1} ; y_{2}=\operatorname{arctanh} m_{0}
$$

The solutions of (35) and (36) are the same, but still, the processing and its speed might be different due to different solutions density and location relatively to the axes - Figure 3. Before building the 

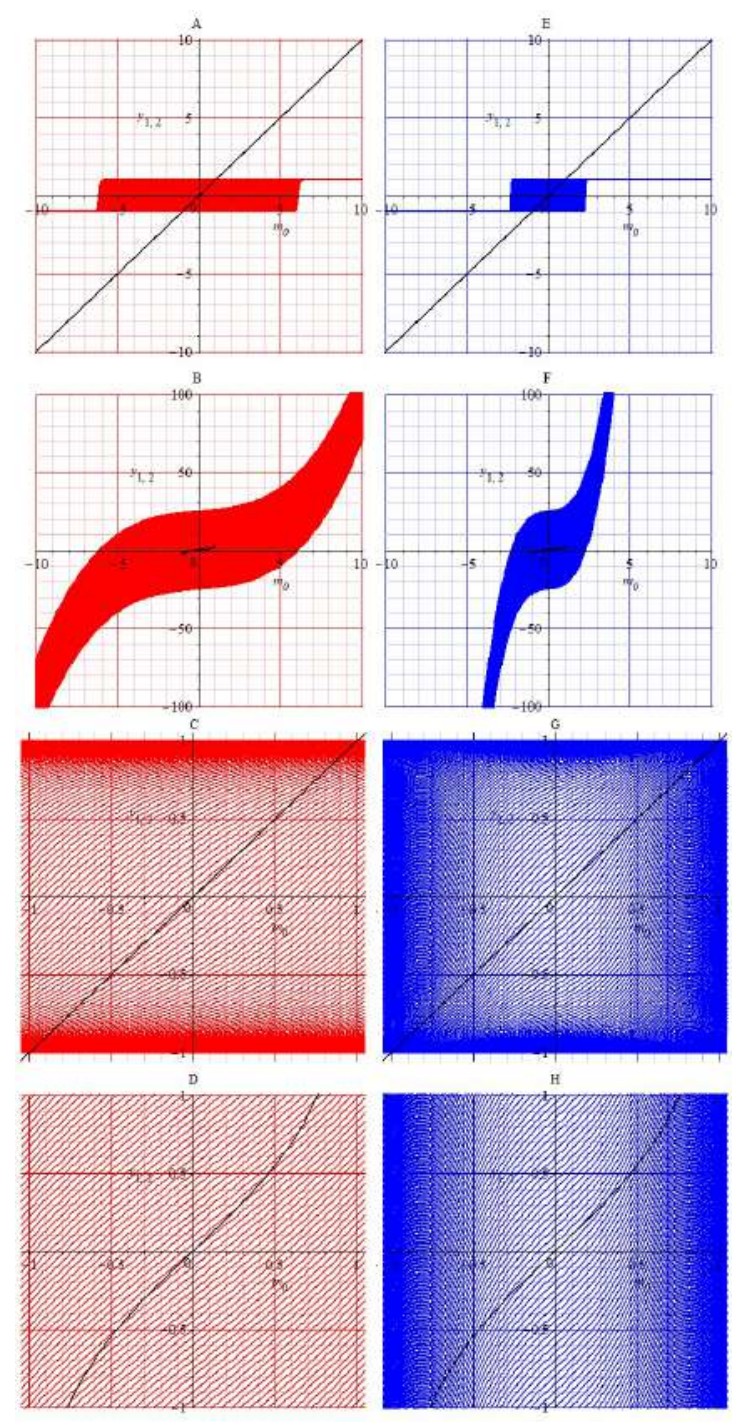

Figure 3. Functions sets general mathematical behavior (for arbitrary element), represented by the corresponding curves, with changes of two parameters. $\alpha=-50 . .50 . \alpha$ increases in 0.1. The plot contains 1001 curves (marked with red and blue colors), each intersects with black curves. (A), (B), (C), (D): $T=1400[K]$. (E), (F), (G), (H): $T=300[K]$. (A), (C), (E), (G): (35). (B), (D), (F), (H): (36). As the result, with the temperature decrease, the sets of curves contract, producing multiple intersections

connection with physics, we considered important to study the functions sets general mathematical behavior - in order to find out the consequence of functions sets behavior and properties.

According to Figure 3 we conclude, that with applying the first system (35) the functions set thickens in one point, while with applying the second system (36), the functions set thickens along the straight line, parallel to $y_{1,2}$ axis. It makes the difference in data processing speed and quality. We recommend both variants to be used, and then to choose the result of better processing in building magnetization patterns compared by the computation time and visible quality of the resulting patterns.

For the majority of tasks, including mean magnetization per electron, we imply the change of $\alpha$ in limits $\alpha=-2.2$, where its values increase in 0.1 for each solution. In general, there are $41 \alpha$ values, respectively -41 curves of tanh $\xi$. It imitates the changing external magnetic field. Temperature remains constant and its value is set manually at the beginning. Figure 3 illustrates the amplification of curves number in order to emphasize the thickening, but in concrete array creation 41 curves are fully enough in magnetic saturation catching. Saturation - the region, where the magnetization pattern is constant relatively to $m_{0}$ axis. Arrays in $\alpha$-change are automatically generated by applying the next 


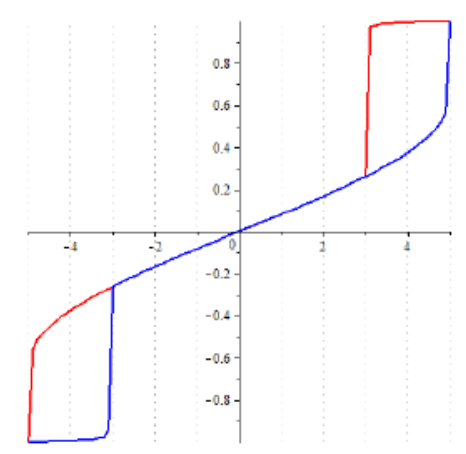

Figure 4. $m_{0}(\alpha)$. Algorithmic built double loop for Cobalt $(T=300[K], J$ is evaluated by (22)). Double loops are presented as a defined prospective of Heisenberg model development for complicated hysteresis cases, look [31].

commands: Appendix, Figure 28. Similar with Figure 25, in Figure 28 (computational details are given again at Appendix) firstly comes the borders creation - two integer numbers, denoting the index of each future value (A).

As it is observable from Figure 1 and Figure 3, hysteresis loops originate in case of multiple intersections of $Q 0$ with $Q 2$ (35), or $Q 4$ with $Q 6$ (36). There are various possible intersection patterns: single point, two, three, four or five points. The single point gives a smooth curve pattern, while multiple intersections produce the variety of loops. If all multiple intersections of each set curve occur at one quarter, but the central part has single intersections, we obtain double loops as in Figure 4. This result takes place at low temperatures $(T \leq 300[K])$, see also [31].

\subsection{Mean per electron magnetization visualization}

After applying a numeric evaluation, we can built ordinary curves by converting two lists into the axes of a plot (see Figure 33 at Appendix ). With the decrease of temperature systems start intersecting more times, producing curves. Low temperature range - lower than Curie pointshows a ferromagnetic pattern behavior, high temperature range - higher than Curie point - shows a paramagnetic behavior. That is why the separate algorithmic part is dedicated to paramagnetic patterns at high temperature range.

Going to the ferromagnetism domain, for loops building we firstly need to analyze and transform the data in terms of the variable $\alpha$. The changes are to be done with solution values. This time the intersections are rewritten in a new array, as it would allow to assign several values to one index number. This array is transformed into the array of sets by convert(array $[i]$, set), where $i$ is the array index number. Then, the method of maximums and minimums separation is made by $\max (\operatorname{set}[i])$ and $\min (\operatorname{set}[i])$ as at the Appendix, Figure 32, It makes each set of values to be dissected: from overall index set the maximum and the minimum values are rewritten in separate lists.

The smoothness of obtained hysteresis loops is estimated as well - Figure 33 at Appendix. As we can see, the way of patterns rising is directly determined by material thermal energy change, namely - by temperature change. The size of a coercive force in $\alpha$ units is directly connected with material parameters (number of closest neighbors $z$, exchange integral $J$ ) and temperature too. The pattern of loop bifurcation starting point (as it is shown for Nickel - Appendix - Figure 33) is connected with critical temperature point - Curie point $\theta$.

Resulting hysteresis patterns by Heisenberg theory are more rectangular (showing hard magnetization patterns), that is why without additional coefficients implication for their deformation, they could be adapted for descriptions of some micro-bodies [32], for example: nanowires [33] and nanothreads, or groups of materials, which are thermally annealed in magnetic field (p. 856, [34]), as their patterns have rectangular nature as well as the models by Heisenberg theory have. With the increase of temperature, magnetization hardness gets reduced - Figure 6. 


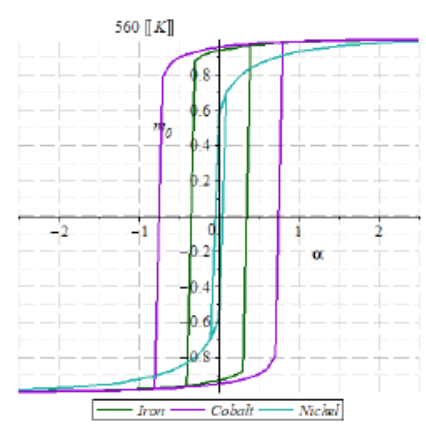

Figure 5. Iron deviance reduction by $z_{\mathrm{Fe}}=9$ assumption instead of physically reasoned $z_{\mathrm{Fe}}=8$ (Section 2). It is done by the means of mathematical selection only.

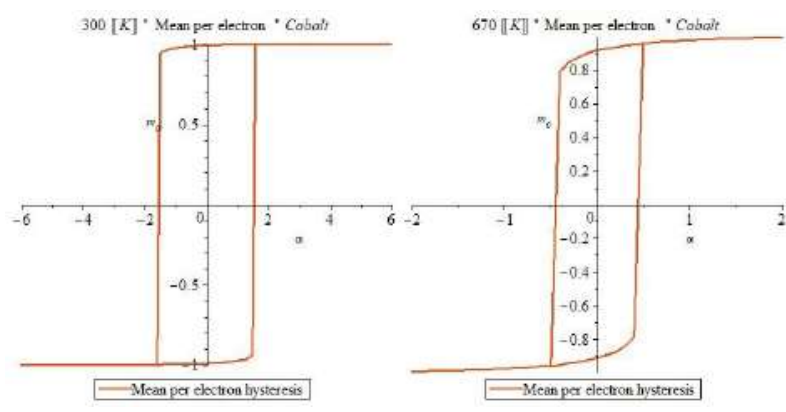

Figure 6. Loops decay smoothness comparison for Cobalt. Left pattern - $T=300[K]$, the coercive force $\alpha=1.6$. Right pattern $-T=670[K]$, the coercive force $\alpha=0.45$.

As the coercive force - the value of magnetic field strength, needed for full material demagnetization - is already mentioned in estimations, let us proceed to the curve of primary material magnetization [1]. Before obtaining the pattern of remanent magnetization - our focus, the material should be magnetized with external magnetic field application, to move the internal material magnetization from zero [6]. Primary curve of material magnetization is built by Heisenberg expressions - Figure 7. Its pattern is built by the same methods as ordinary curves in paramagnetic cases.

\section{Multi-layer nanoparticle magnetization}

A nanoparticle is the most important object for our investigations, because for small enough dimension of a body the domain structure is most simple: it is one-domain case, so it is the main object for the Heisenberg model $[35,36]$. When we go down in the dimension, another question arises, a contribution of surface in magnetic properties. A multi-layer model may give an answer for the posed question, establishing the border of surface phenomena significance. In the case of physical tasks for larger bodies, it is used to omit the surface phenomena due to its small impact [9]; i.e. if the scale of an object is bigger than thousands of nanometers. Vice versa, if the scale of an object is of nanoscale order, surface phenomena is considered to be accounted [8]. During the developments we divided a spherical model of a nanoparticle into several layers, and then fixed, when the surface phenomena (in our case - exchange interaction between nanoparticle atoms) causes the impact on magnetization pattern. A nanoparticle is divided into the layers by the exchange interaction factor, that is why new layers were not subtracted from the previous.

\subsection{Parameters computation for two-layer and three-layer nanoparticles}

For nanoscale magnetism studies we regarded working with spherical nanoparticles, as their shape is the most convenient for primary estimations due to its uniform central symmetry - Figure 8 . In defining the number of active magnetic electrons we adapted a common formula for a spherical 


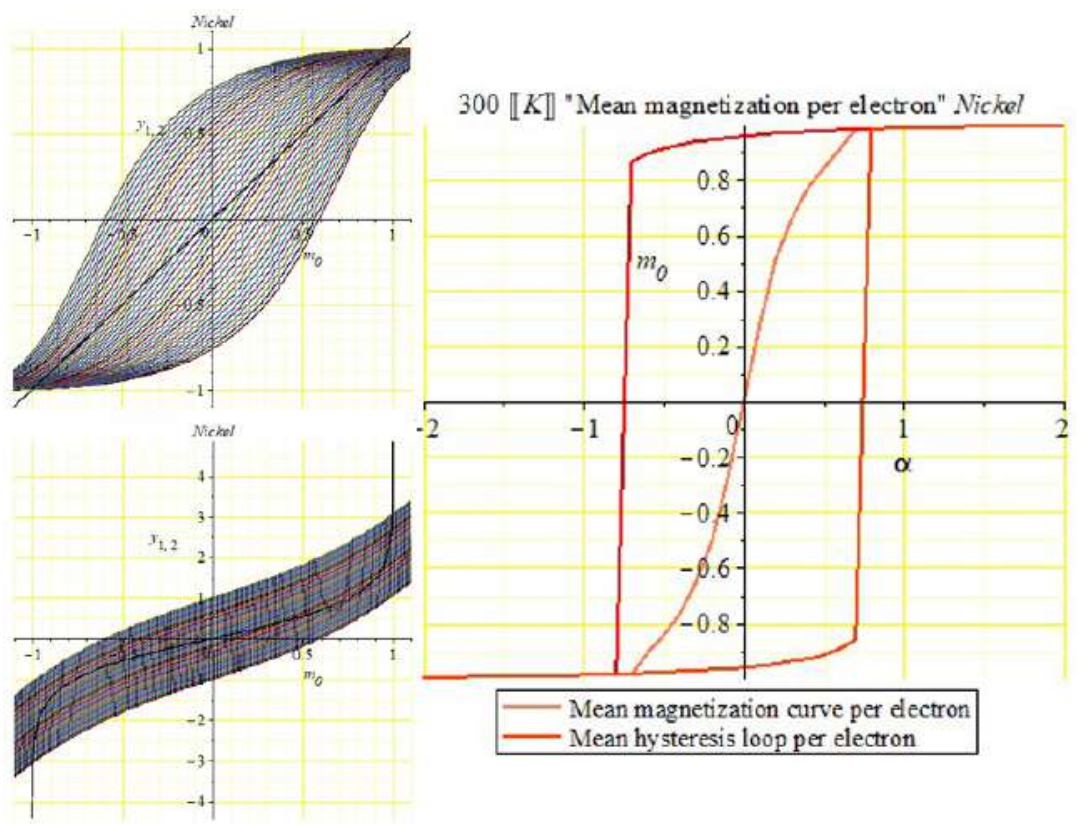

Figure 7. Primary magnetization, located in a center of a loop. It is defined simultaneously with a hysteresis loop by $f$ solve $(x)$ command application. It computes single-valued list for each index, regardless if exists more intersections than one.

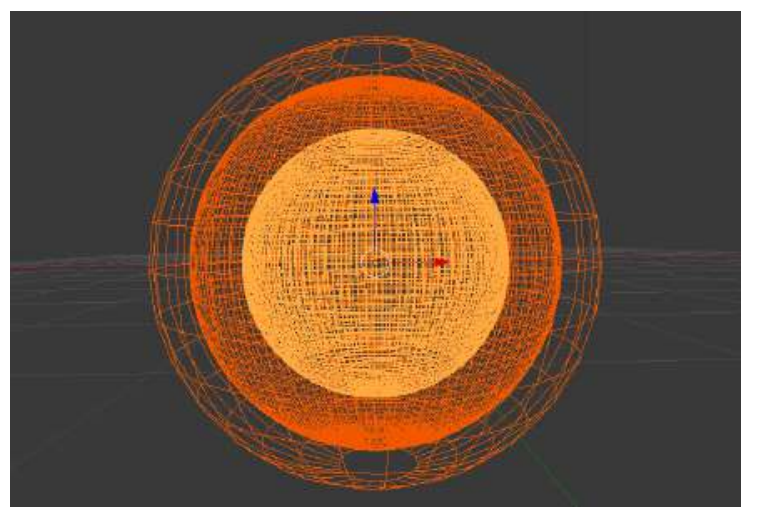

Figure 8. Space spherical division. As it is shown, the grids represent the order of layer division. The yellow grid shows the inner sphere, which is accounted homogeneous for its parameters values. The space inside the thick orange grid is segregated, when we define the second layer. The space inside the rare orange grid is additionally segregated, when the third layer is defined. Without any layer segregation, phenomena from space outside yellow grid are omitted.

shell volume. It is the representation of an annulus in a three-dimensional case [9], defined as the subtraction of the smaller nested sphere with radius $r_{2}$ from the bigger total sphere with radius $r_{1}$, for its volume we write:

$$
V=\frac{4}{3} \pi r_{1}^{3}-\frac{4}{3} \pi r_{2}^{3}
$$

We considered two main configurations of nanoparticle models useful in nanoscale physics: a two-layer spherical nanoparticle - Figure 9, and a three-layer spherical nanoparticle - Figure 10. Nonetheless, more number of layers can be distinguished using the same principles, illustrated throughout this section. In preparing the number of electrons parameter $n$, we come to calculation of each atomic layer consistency.

Each additional layer has the thickness, equal to the thickness of a mono-atomic layer. The approximate diameter of each element atom is taken as an elementary layer unit by $d=2 r$, where $r-$ atom radius. Each element value is represented in Table 3. 
Table 3. Element radii $r$ values [9]

\begin{tabular}{|l|l|l|}
\hline Element & Radius in $[\mathrm{pm}]$ & Radius in $[\mathrm{m}]$ \\
\hline Iron & 156 & $156 \cdot 10^{-12}$ \\
\hline Nickel & 149 & $149 \cdot 10^{-12}$ \\
\hline Cobalt & 152 & $152 \cdot 10^{-12}$ \\
\hline
\end{tabular}
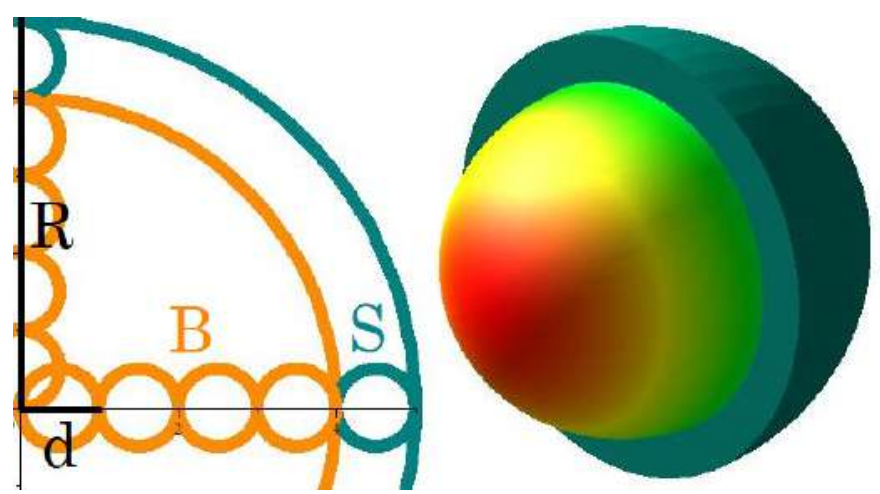

Figure 9. Two-layer nanoparticle simplification. Number of electrons defining. $\mathrm{R}$ - whole nanoparticle radius, $\mathrm{d}$ - element node diameter. $\mathrm{B}$ - bulk layer, $\mathrm{S}$ - surface layer.
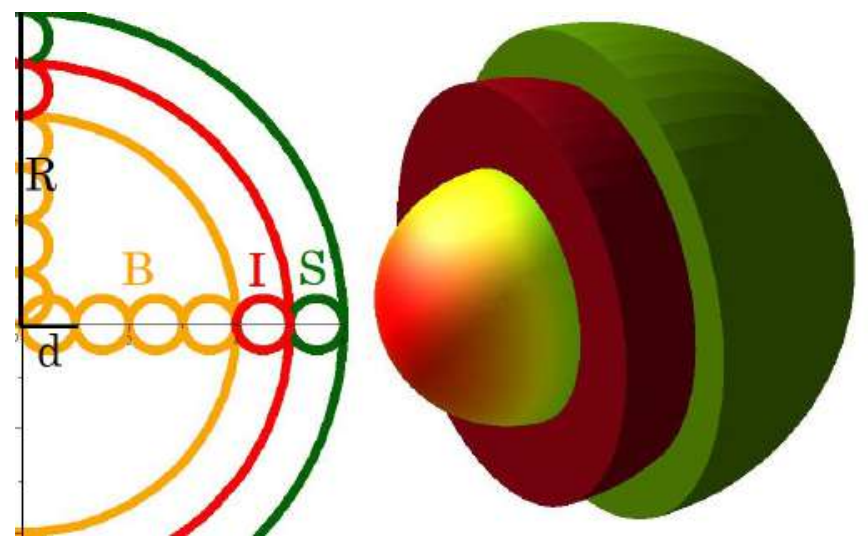

Figure 10. Three-layer nanoparticle model. Number of electrons defining. $\mathrm{R}-$ whole nanoparticle radius, $\mathrm{d}$ - element node diameter. $\mathrm{B}$ - bulk part, I - intermediate part, $\mathrm{S}$ - surface part.

We have obtained needed visual information about particles structure and their size parameters from Figure 9 and Figure 10. $R$ is a total nanoparticle radius. Moreover, we can establish, that in implying new layers we just exhale them without additional transformations of previously modeled ones. There are three number of electrons values, separate for each layer: $n_{B}$ - number of electrons in a bulk part, located in the center of a particle, $n_{I}$ - number of electrons in an intermediate layer with the thickness of mono-atomic layer, $n_{S}$ - number of electrons in a surface layer with the thickness of mono-atomic layer. They are derived transforming (37) for our case:

$$
\begin{gathered}
n_{B}=\frac{\frac{4}{3} \pi(R-d)^{3}}{d^{3}}=\frac{\frac{4}{3} \pi R^{3}-4 \pi R^{2} d+4 \pi R d^{2}-\frac{4}{3} \pi d^{3}}{d^{3}}, \\
n_{I}=\frac{4}{3} \pi \frac{(R-d)^{3}-(R-2 d)^{3}}{d^{3}}, \\
n_{S}=\frac{4}{3} \pi \frac{R^{3}-(R-d)^{3}}{d^{3}}=4 \pi \frac{R^{2}-R d+\frac{1}{3} d^{2}}{d^{2}} .
\end{gathered}
$$

Then, the most suitable nanoparticle size is established numerically - Table 4. Concluding the calculations for Iron two-layer model, we can make an inference, that the most relevant nanoparticle 
Table 4. Number of electrons comparison for Iron two-layer nanoparticle of various sizes

\begin{tabular}{|l|l|l|l|l|l|l|l|}
\hline $\mathrm{R}$ in $[\mathrm{nm}]$ & 1 & 3 & 5 & 10 & 15 & 50 & 100 \\
\hline $\mathrm{R}$ in $[\mathrm{m}]$ & $1 \cdot 10^{-9}$ & $3 \cdot 10^{-9}$ & $5 \cdot 10^{-9}$ & $1 \cdot 10^{-8}$ & $1.5 \cdot 10^{-8}$ & $5 \cdot 10^{-8}$ & $1 \cdot 10^{-7}$ \\
\hline$n_{S}$ & 93 & 1045 & 3030 & $1.25 \cdot 10^{4}$ & $2.84 \cdot 10^{4}$ & $3.2 \cdot 10^{5}$ & $1.28 \cdot 10^{6}$ \\
\hline$n_{B}$ & 44 & 2678 & 14209 & $1.25 \cdot 10^{5}$ & $4.37 \cdot 10^{5}$ & $1.69 \cdot 10^{7}$ & $1.36 \cdot 10^{8}$ \\
\hline
\end{tabular}
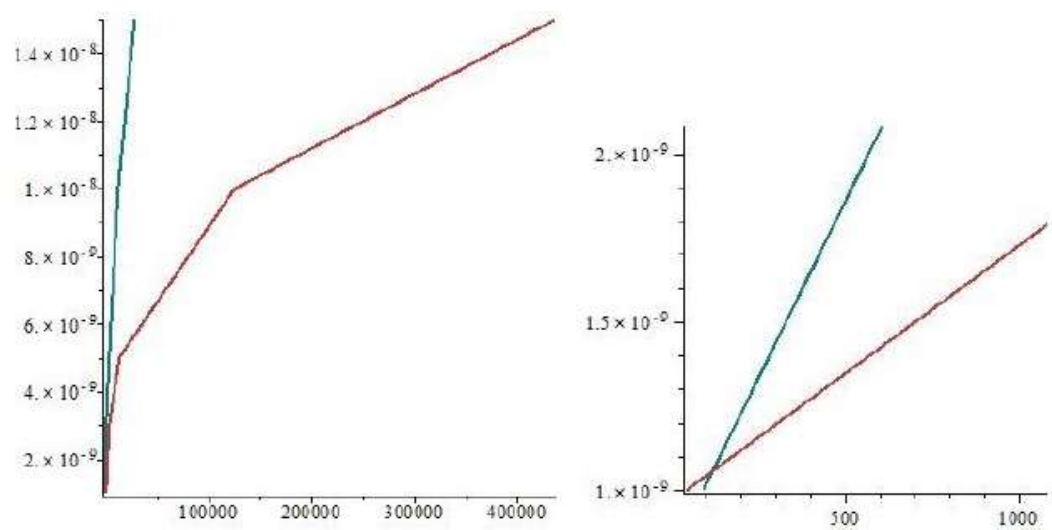

Figure 11. Establishing the smallest border of nanoparticle multilayer model workability. Blue monoatomic layer atoms number, red - central part atoms number. $\mathrm{X}$ axis - atoms number, $\mathrm{Y}$ axis nanoparticle radii. Left and right plots differ in the atoms number range.

size for our modeling is with $5 \mathrm{~nm} \geq R \geq 3 \mathrm{~nm}$. With decreasing the radius up to 1 nanometer, the surface layer becomes thicker than the bulk layer - in this case film models are to be applied instead of bulk. As for the bigger radius - it lesser causes the impact, that is why up to 100 nanometers it gradually becomes inconspicuous - Figure 11.

The next, microscopic, parameters for nanoparticle division: exchange integral $J$, number of closest neighbors $z$, and the combined material parameter, composed from $J, z$ and thermal energy $k T$. The last constitutes general material characteristics, as it was mentioned in the Curie magnetization section. The evaluation of these group of parameters is done sequentially on the ground of physical reasons.

Analyzing Figure 12, we observe the patterns of exchange interactions parameters. Where the lines are thicker, the interaction is stronger. As the interaction patterns of a bulk layer (marked red) fully cover the whole nanoparticle, the exchange integral remains the same as in previous model of mean magnetization per electron. So we just use the expression, which was primary derived in Curie region section:

$$
J_{B}=\frac{k \theta}{2}\left(1 \pm \sqrt{1-\frac{8}{z_{B}}}\right) .
$$

It is evaluated through the concrete values; $z_{B}$ is the same as $z$ from mean per electron model. However, both monoatomic layer exchange patterns (marked blue) and corresponding exchange integrals require changes. We supposed that the values of exchange integral increases with approximate percentage ratio. For intermediate layer its value is bigger in 0.15 , in surface layer - in 0.25 .

$$
J_{I}=J_{B} \cdot 1.15 ; J_{S}=J_{B} \cdot 1.25 \text {. }
$$

Analogically it is made for number of closest neighbors for each part, implying the same command of discarding non-integer numbers parts as in Figure 34 of Appendix. The difference is in other percentage relation:

$$
z_{I}=z_{B} \cdot 0.75 ; z_{S}=z_{I} \cdot 0.78,
$$




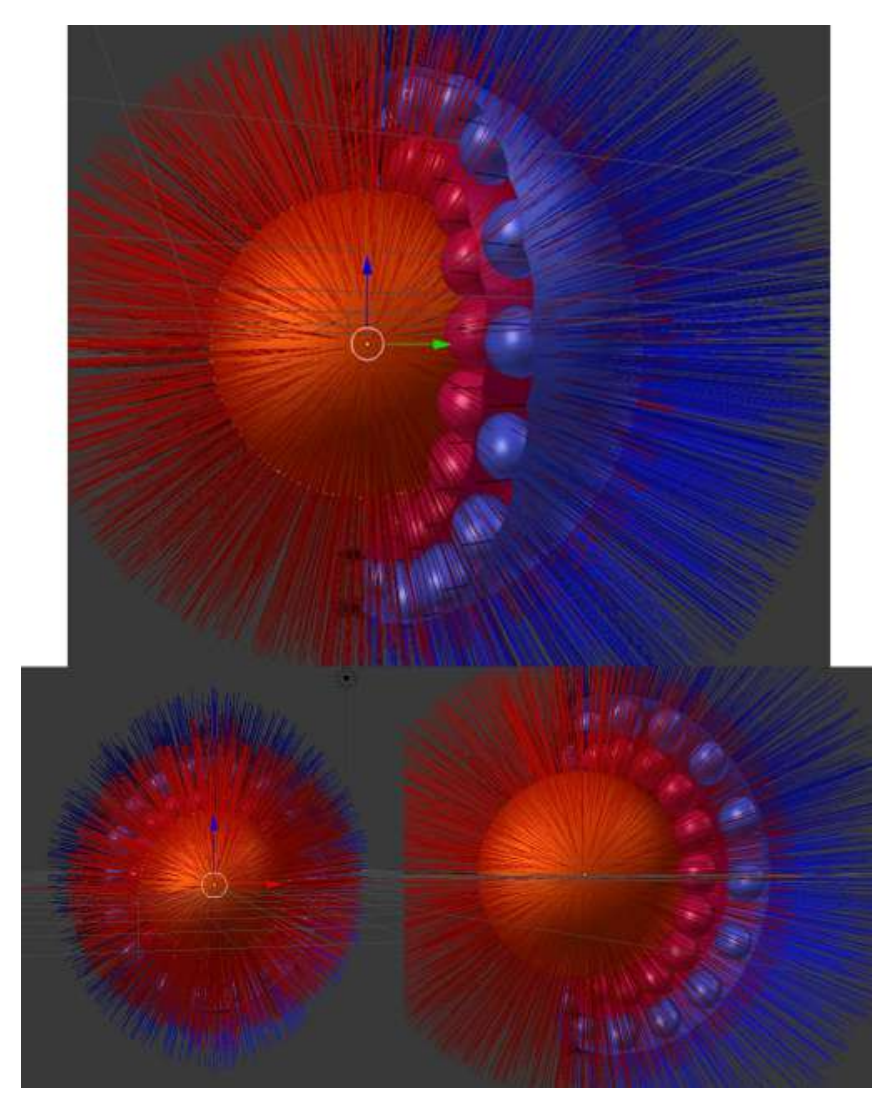

Figure 12. Exchange interactions estimation. Red lines - the internal exchange parameter. Blue lines - the surface exchange parameter. Spheres - nodes, representing each layer. The more outer node occupies the atom, the less its exchange interaction is compensated. For all edge nodes exchange interactions occur from one side only. That is why the exchange integral $J$ changes at each layer.

where intermediate layer contains 0.75 of the bulk one, while surface layer contains 0.78 of intermediate. The percentage in this case is selected to obtain homogeneous decrease of each with accounting the crystal structure of each material, see Figure 23 and Figure 24 at Appendix. The parameter $\beta$ is just separated for each layer $-\beta_{B}, \beta_{I}, \beta_{S}$, and $z_{B}, z_{I}, z_{S}$ evaluation results are inserted in its evaluation:

$$
\beta_{B}=\frac{z_{B} J_{B}}{k T} ; \beta_{I}=\frac{z_{I} J_{I}}{k T} ; \beta_{S}=\frac{z_{S} J_{S}}{k T} .
$$

The results of $z, J$ and $\beta$ particular evaluation are represented in Figure 35 for Cobalt mono-elemental nanoparticle (Appendix).

\subsection{Partition functions for two-layer and three-layer nanoparticles}

When all the values are computed and the connection between them is automatically set for each case and material, we are to highlight the energy distribution for multilayered nanoparticle and derive the partition function for every layer.

Let the interaction between layers is neglected, except the contributions in neighbours numbers and exchange integrals value, supposing that the total Hamiltonian of the whole body is just the sum of Hamiltonians of each layer. Under such assumption the partition function can be separated for both models $\left(Z_{I I}\right.$ and $Z_{I I I}$ - index II for a two-layer nanoparticle, index III for a three-layer nanoparticle), using the property of exponent $e^{a+b}=e^{a} \cdot e^{b}$ :

$$
\exp \left[-\frac{\hat{\mathcal{H}}_{\mathcal{I I}}}{k T}\right]=\exp \left[-\frac{\hat{\mathcal{H}}_{\mathcal{B}}}{k T}-\frac{\hat{\mathcal{H}}_{\mathcal{S}}}{k T}\right]=\exp \left[-\frac{\hat{\mathcal{H}}_{\mathcal{B}}}{k T}\right] \cdot \exp \left[-\frac{\hat{\mathcal{H}}_{\mathcal{S}}}{k T}\right]
$$


where $\hat{\mathcal{H}}_{\mathcal{I I}}$ is the Hamiltonian of a two-layer nanoparticle. Hence

$$
Z_{I I}=Z_{B} Z_{S}
$$

and, after implying both of substitutions into Gibbs operator general definition (1), we obtain

$$
\frac{1}{Z_{I I}} \exp \left[-\frac{\hat{\mathcal{H}}_{\mathcal{I I}}}{k T}\right]=\frac{1}{Z_{B} Z_{S}} \exp \left[-\frac{\hat{\mathcal{H}}_{\mathcal{B}}+\hat{\mathcal{H}}_{\mathcal{S}}}{k T}\right]
$$

It leads to the Gibbs operator expression for two layers $\hat{g}_{w I I}$ (during this section index "w" is used for denoting energy levels instead of canonical " $n$ ", in order to avoid mixing up with denotation of electrons number " $n ")$ :

$$
\hat{g}_{w I I}=\hat{g}_{w B} \cdot \hat{g}_{w S}=\frac{1}{Z_{B} Z_{S}} \exp \left[-\frac{\hat{\mathcal{H}}_{\mathcal{B}}+\hat{\mathcal{H}}_{\mathcal{S}}}{k T}\right]
$$

defining energy spectra for each layer, when Hamilton operator is already applied

$$
Z_{B}=\sum_{w} \exp \left[\frac{E_{w B}}{k T}\right] ; Z_{S}=\sum_{w} \exp \left[\frac{E_{w S}}{k T}\right]
$$

Here $E_{w B}$ - energy spectrum for a bulk layer, $E_{w S}$ - energy spectrum for a surface layer.

For defining three layers operators we do alike transformations, transforming the general definition (1) into

$$
\frac{1}{Z_{I I I}} \exp \left[-\frac{\hat{\mathcal{H}}_{\mathcal{I I I}}}{k T}\right]=\frac{1}{Z_{B} Z_{I} Z_{S}} \exp \left[-\frac{\hat{\mathcal{H}}_{\mathcal{B}}+\hat{\mathcal{H}}_{\mathcal{I}}+\hat{\mathcal{H}}_{\mathcal{S}}}{k T}\right] .
$$

As the result, the Gibbs distribution expression for three layers $\hat{g}_{w I I I}$ is

$$
\hat{g}_{w I I I}=\hat{g}_{w B} \cdot \hat{g}_{w I} \cdot \hat{g}_{w S}=\frac{1}{Z_{B} Z_{I} Z_{S}} \exp \left[-\frac{\hat{\mathcal{H}}_{\mathcal{B}}+\hat{\mathcal{H}}_{\mathcal{I}}+\hat{\mathcal{H}}_{\mathcal{S}}}{k T}\right] .
$$

The connection of the energy spectra with partition function for each layer is given by

$$
Z_{B}=\sum_{w} \exp \left[\frac{E_{w B}}{k T}\right] ; Z_{I}=\sum_{w} \exp \left[\frac{E_{w I}}{k T}\right] ; Z_{S}=\sum_{w} \exp \left[\frac{E_{w S}}{k T}\right],
$$

$E_{w B}$ - energy spectrum for a bulk layer, $E_{w I}$ - energy spectrum for an intermediate layer, $E_{w S}$ - energy spectrum for a surface layer. Let us proceed to the connection of obtained transformations. All the values are included in $\xi_{B}, \xi_{I}$ and $\xi_{S}$, that looks identically, for example,

$$
\xi_{B}=\frac{\alpha+\beta_{B} \frac{m_{0}^{[B]}}{n_{B}}-\beta_{B}^{2} \frac{m_{0}^{[B]}}{n_{B} z_{B}}+\beta^{2} \frac{\left(m_{0}^{[B]}\right)^{3}}{2\left(n_{B}\right)^{3} z_{B}}}{2} .
$$

Hence, the partition function for the corresponding layer reads

$$
Z_{B}=K\left[2 \cosh \xi_{B}\right]^{2 n_{B}} ; Z_{I}=K\left[2 \cosh \xi_{I}\right]^{2 n_{I}} ; Z_{S}=K\left[2 \cosh \xi_{S}\right]^{2 n_{S}} .
$$

On this ground, for magnetization variable $m_{0}$ we have:

$$
m_{0}^{[B]}=\frac{\partial \ln Z_{B}}{\partial \alpha}=\frac{\partial \ln \left(K\left[2 \cosh \xi_{B}\right]^{2 n_{B}}\right)}{\partial \alpha},
$$


the corresponding $m_{0}^{[I]}, m_{0}^{[S]}$ looks similar. For two-layer spherical nanoparticle the expression (16) changes the form with substituting the partition function $Z_{2}$ :

$$
\begin{gathered}
m_{0}^{[B S]}=\frac{\partial \ln Z_{2}}{\partial \alpha}=\frac{\partial \ln Z_{B} Z_{S}}{\partial \alpha}=\frac{\partial \ln Z_{B}}{\partial \alpha}+\frac{\partial \ln Z_{S}}{\partial \alpha}=\frac{\partial \ln \left(K\left[2 \cosh \xi_{B}\right]^{2 n_{B}}\right)}{\partial \alpha}+\frac{\partial \ln \left(K\left[2 \cosh \xi_{S}\right]^{2 n_{S}}\right)}{\partial \alpha}= \\
=n_{B} \tanh \xi_{B}+n_{S} \tanh \xi_{S} .
\end{gathered}
$$

As the result, the expression for $m_{0}^{[B S]}$ is derived analogically as in Section 4 , for mean magnetization per electron model. The difference is in number of electrons impact. For three-layer spherical nanoparticle the expression (16) changes the form with substituting the partition function $Z_{3}$ :

$$
\begin{gathered}
m_{0}^{[B I S]}=\frac{\partial \ln Z_{3}}{\partial \alpha}=\frac{\partial \ln Z_{B} Z_{I} Z_{S}}{\partial \alpha}=\frac{\partial \ln Z_{B}}{\partial \alpha}+\frac{\partial \ln Z_{I}}{\partial \alpha}+\frac{\partial \ln Z_{S}}{\partial \alpha}= \\
=n_{B} \tanh \xi_{B}+n_{I} \tanh \xi_{I}+n_{S} \tanh \xi_{S},
\end{gathered}
$$

which demonstrates the possibility of multiple inserting of new layers, if to compare $m_{0}^{[B I S]}$ derivation procedure with the derivation for $m_{0}^{[B S]}$. In building the resultant magnetization patterns, the system of equations from mean magnetization per electron model (35) is changed into the pairs of systems like this:

$$
y_{1}=n_{B} \tanh \xi_{B} ; y_{2}=m_{0}^{[B]} .
$$

The alternative variant, like (36), which is easier for data processing, writes, e.g. as:

$$
y_{1}=\xi_{B} ; y_{2}=\operatorname{arctanh}\left(\frac{m_{0}^{[B]}}{n_{B}}\right) .
$$

where $m_{0}^{[B]}, m_{0}^{[I]}, m_{0}^{[S]}$ are the variables of different order. Data from each system solution is added by adding each index value $[j]$ of one layer to the correspondent index value of the other layers. Every index is marked identically and every array has to have the same number of index values.

$$
\begin{gathered}
m_{0}^{[B S]}[j]=m_{0}^{[B]}[j]+m_{0}^{[S]}[j], \\
m_{0}^{[B I S]}[j]=m_{0}^{[B]}[j]+m_{0}^{[I]}[j]+m_{0}^{[S]}[j] .
\end{gathered}
$$

The resulting sums $m_{0}^{[B S]}[j]$ and $m_{0}^{[B I S]}[j]$ are the total magnetization patterns of a two-layer nanoparticle and a three-layer nanoparticle. They give us the concrete review of mono-atomic layers impact in total nanoparticle magnetization of the given size.

\subsection{Magnetization curves and hysteresis loops for two-layer and three-layer nanoparticles}

\subsubsection{Paramagnetic case}

The starting point of a multilayer spherical nanoparticle modeling is in defining $m_{0}^{[B]}[j]$. The principles and methods are the same as in the mean per electron model, but have some peculiarities, highlighted here. The paramagnetic case for a Cobalt ( 6 nanometer-sized particle, $R=3[\mathrm{~nm}]$ ) is considered to be the example for a high-temperature modeling part. $T=1200[\mathrm{~K}]$. A bulk layer is firstly created separately - Figure 36 at Appendix.

Though, multiple solutions could occur only in a bulk part, that is why loop occurrence checking is done for a bulk layer only. There is given the reason of hysteresis occurrence impossibility. Mono-atomic layers parameters, such as exchange integrals $J$, number of closest neighbors $z$ and each layer thickness have different values from a bulk one, denoting less compensated exchange 


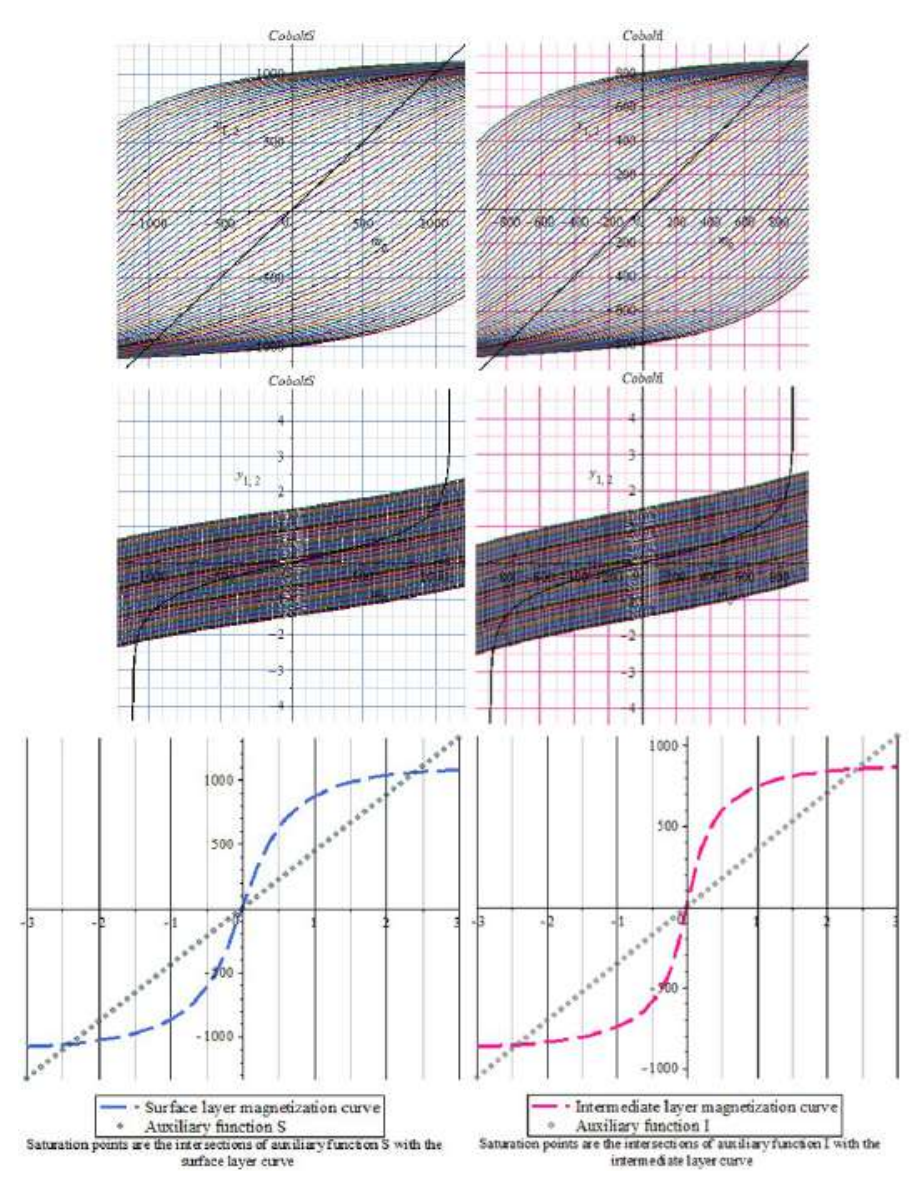

Figure 13. Paramagnetic surface (blue) and intermediate (pink) magnetization patterns creation. Auxiliary functions show each saturation beginning by intersecting the correspondent curves. Comparatively with bulk pattern (Figure 36, Appendix) paramagnetic S and I patterns are always smooth out of parameters peculiarity condition.

interactions. The position of atoms in nodes of intermediate and surface layers do not give needed curvature for multiple intersections of functions sets.

Despite the fact that intermediate and surface layers have the same thickness, peculiarities of exchange interactions lead to the consequence in patterns difference. In any case the surface layer pattern is less curved, than the intermediate one. On the other hand, the number of electrons in surface part is always bigger, which produces greater impact in total nanoparticle magnetization - Figure 13.

All auxiliary functions are calculated for each curve through the auxiliary function multiplier $\eta=0.04$, which is defined through the percentage ratio. It is multiplied on particular layer number of electrons $n$ and each value index $j$. The resultant list of values is discrete, which helps us not only in saturation defining, but in magnetization patterns smoothing, which is done further in ferromagnetic occurrence sample. Analogical functions can be defined from material peculiarities for particular patterns deformations in other physical situations.

The slope angle of each layer auxiliary function is different, but they show that the saturation region of every layer matches for the whole nanoparticle.

Figure 14 gives the explanation, where ferromagnetic pattern origin is placed at high temperatures region. Para- and ferromagnetic patterns are built here by $f$ solve $(x)$ for ordinary curve and solve $(x)$ with defining maximum and minimum values similarly as in hysteresis case. As we can conclude, both patterns match. 


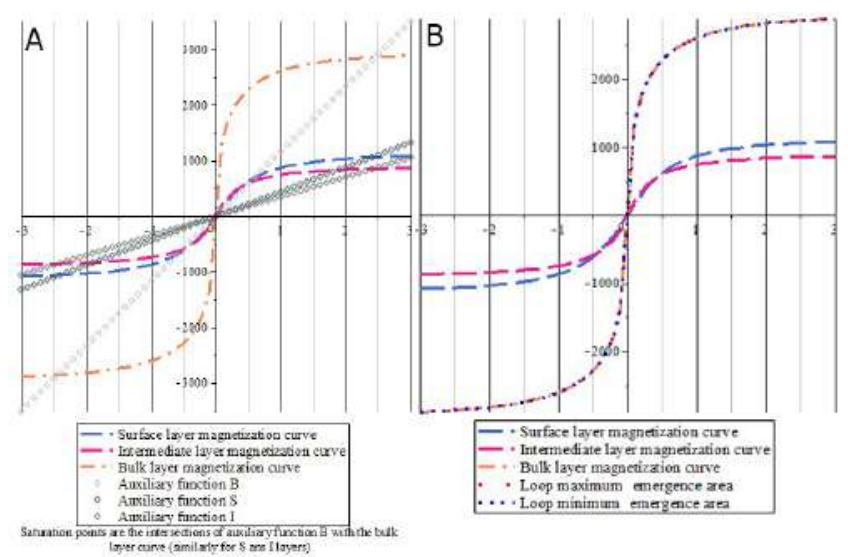

Figure 14. Additional patterns analysis. (A) - Corresponding auxiliary functions help in avoiding incorrect analysis of mono-atomic layers saturation. Comparatively with Figure 13, mono-atomic magnetizations display is deformed by adding the bulk pattern scale. (B) - Loop occurrence area display. It shows, where the magnetization pattern of the whole nanoparticle starts producing hysteresis by reaching ferromagnetic conditions. The paramagnetic pattern and the area of ferromagnetic pattern emergence must match in high temperature range.
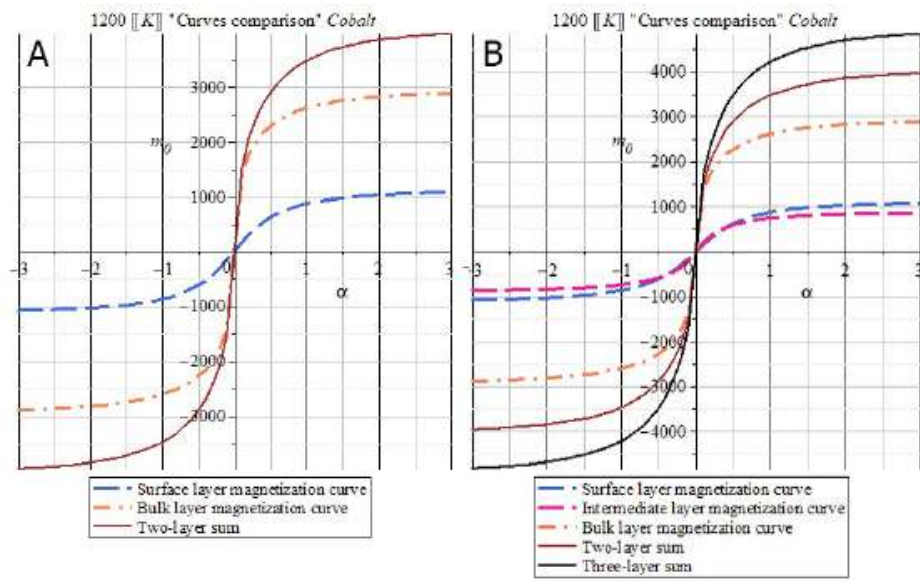

Figure 15. Two-layer and three-layer nanoparticle paramagnetic patterns comparison. Intermediate layer gives a greater impact in total magnetization pattern. Brown curve - two-layer model. Black curve - three-layer model.

Then, the comparison of two-layer and three-layer nanoparticle models is done. As it is described in Figure 15, the intermediate mono-atomic layer gives a significant impact in total nanoparticle magnetization.

Figure 16 proves, that paramagnetic behavior remains the same in temperature slightly upper than the Curie point. On this base we consider this range applicable in practical usage.

If to visualize nanoparticle size studies (Figure 17), a multi-layer model gives a possibility to define mono-atomic layers impact for nanoparticles with various sizes from Table 4, accounting the border of film magnetization emergence from Figure 11. An Iron element is determined applicable in such situation modeling with $z_{B}=8$.

The represented graph shows the border value of a nanoparticle bulk-significant size, as the bulk part starts becoming small relatively to the surface part. In further nanoparticle size decreasing the bulk phenomena disappears, in accordance with the general theoretical background (the exchange interaction is not compensated in films). 


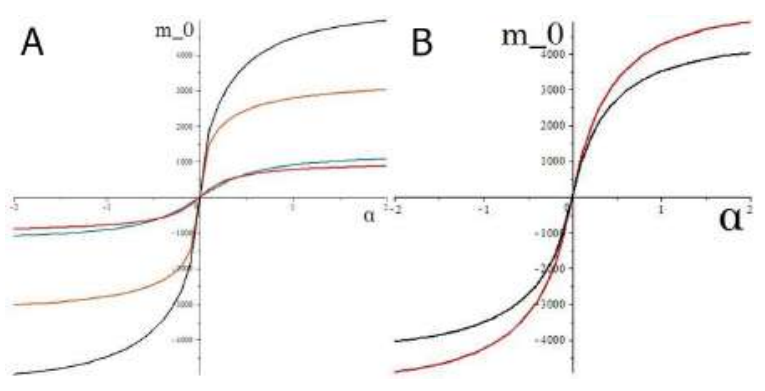

Figure 16. (A) - Three-layer paramagnetic patterns for Nickel nanoparticle $(R=3[n m])$ at $T=660[K]$. Black curve - total nanoparticle magnetization. Orange - bulk. Blue - surface. Pink - intermediate. (B) - Two-layer and three-layer paramagnetic patterns comparison. $T=700[\mathrm{~K}]$. Red - three-layer total magnetization. Black - two-layer total magnetization.
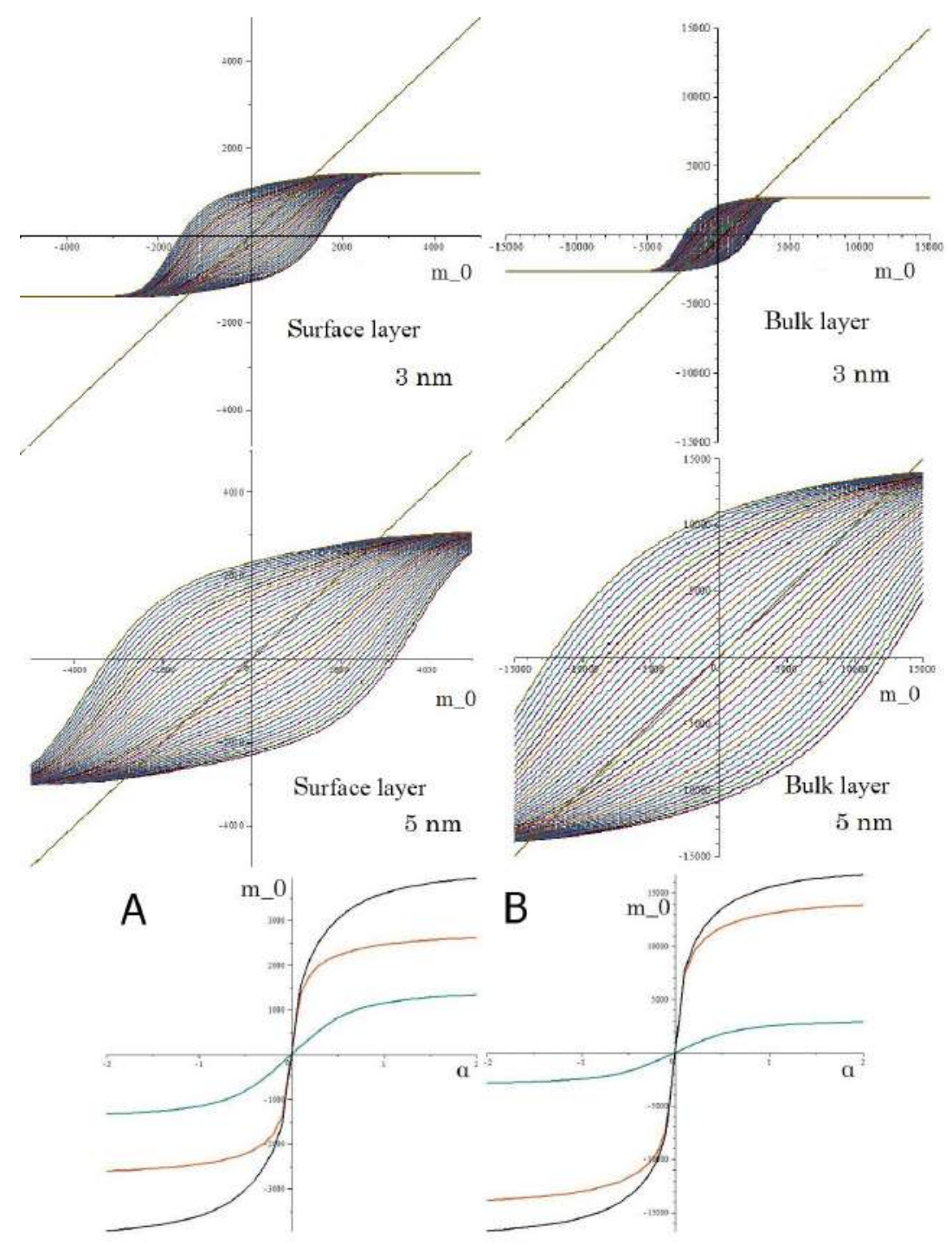

Figure 17. (A) - 6 nanometer-sized $(R=3[\mathrm{~nm}])$ and (B) - 10 nanometer-sized $(R=5[\mathrm{~nm}])$ Iron particles comparison. A two-layer model creation is done. $T=1400[K]$. The surface layer significance vastly differs in a slight nanoparticle size change. The orange curve - a bulk layer impact, the blue curve - a surface layer impact. ' $\mathrm{S}$ ' index - for the surface solution scale, ' $\mathrm{B}$ ' index - for the bulk solution scale. 


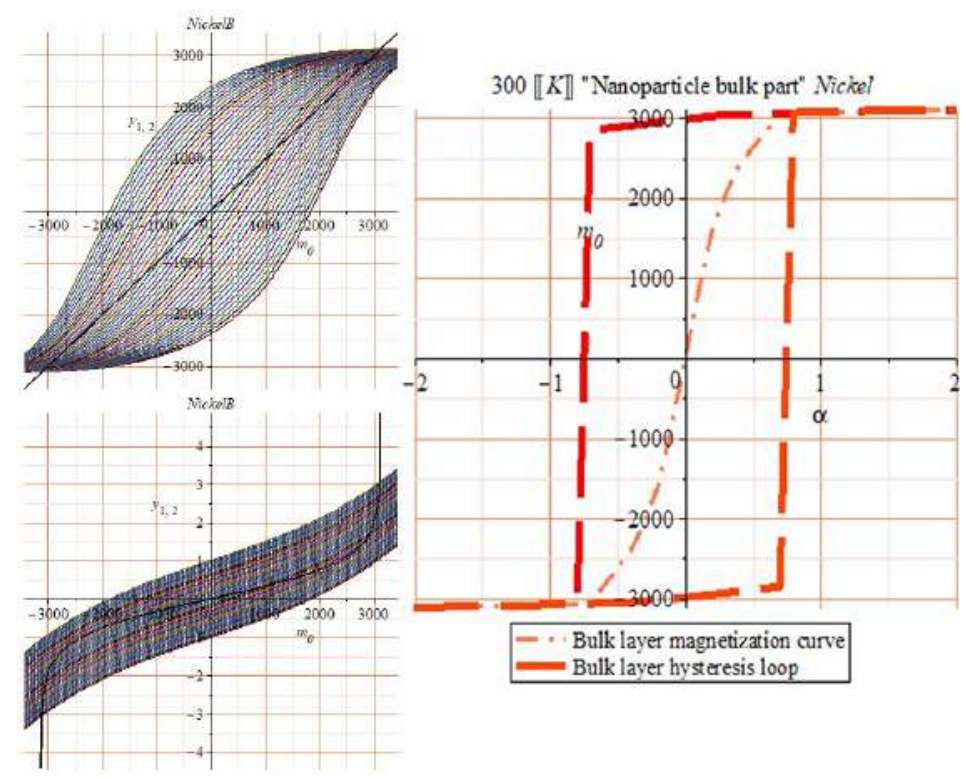

Figure 18. Ferromagnetic bulk magnetization pattern creation. Intersection display and processing result.
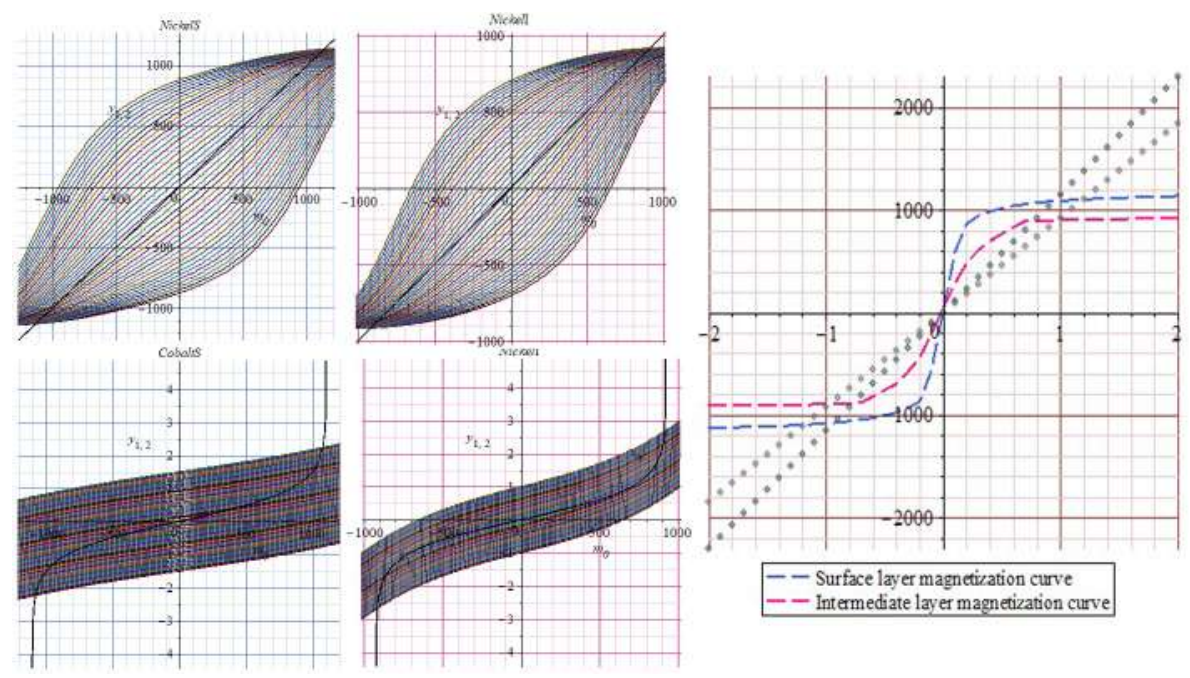

Figure 19. Ferromagnetic surface (blue) and intermediate (pink) magnetization patterns creation. Grey auxiliary functions show each saturation. Patterns are more curved than in paramagnetic case, but still producing single-intersection behavior.

\subsubsection{Ferromagnetic case}

Proceeding to the ferromagnetic pattern derivation, we analogically start with the bulk part magnetization pattern building - Figure 18. The solution is primary made in a nanoparticle scale, though, a large-scaled bulk hysteresis loop is the same as in Figure for mean per electron magnetization. $T=300[K]$. Material - Nickel.

Intermediate and surface ferromagnetic patterns differ noticeably, despite the condition, there is no hysteresis in mono-atomic nanoparticle layers - Figure 19.

In a room temperature there are 1-3 multiple intersecting curves in a central region of intermediate layer. This number is insignificant comparatively with the whole data array and it is not enough for hysteresis loop creation. This fact is considered to be discarded by the virtue of its immateriality, though, intermediate hysteresis generation can be established and accounted with increasing of its layer thickness. 


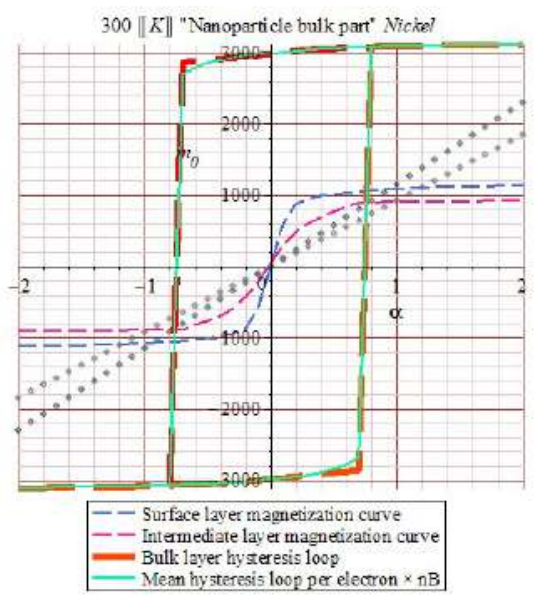

Figure 20. Ferromagnetic magnetization patterns comparison. The mean per electron loop is identical and can be established by multiplying it on number of electrons in a bulk part. This shows the connection of unique isolated magnetization of a single atom and the magnetization of a nanoscale material center.
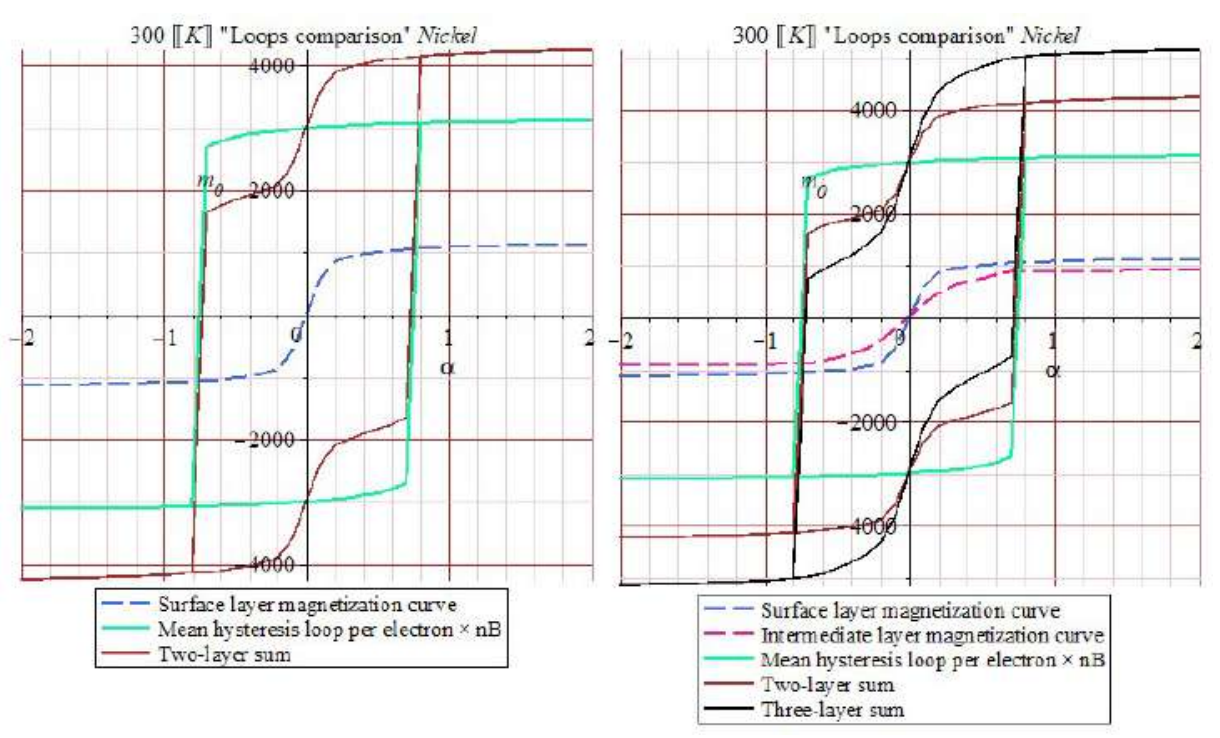

Figure 21. Two-layer and three-layer nanoparticle ferromagnetic patterns comparison. Intermediate layer gives a greater impact in total magnetization pattern. Brown curve - two-layer model. Black curve - three-layer model.

When all the ferromagnetic patterns are defined, let us regard on bulk patterns comparison Figure 20. Due to the low magnitude of processing,the mean magnetization per electron resulting pattern is more smooth and concrete. It allows us to replace the bulk hysteresis pattern with the mean per electron hysteresis.

Multi-layer hysteresis of a spherical nanoparticle is to be compared as well. For this aim Figure 21 is algorithmically built, Figure 22 is made with manual checking to emphasize each separate loop pattern changes. Every layer gives not only the impact in a bulk hysteresis, but the total loop deformation, which can be interpreted as the one of rectangular loop deformation methods.

Heisenberg model combination prospective is traced in Appendix, Sec. 6.2.1. The problems that appears at computational stage of the hysteresis pattern visualisation, such as appearance of prolapses, are posed at Appendix, Subsection 6.5.1, together with subtraction and summation method application, see Subsection 6.5.2. The logic and principles of subtraction checking are sufficiently versatile and its 


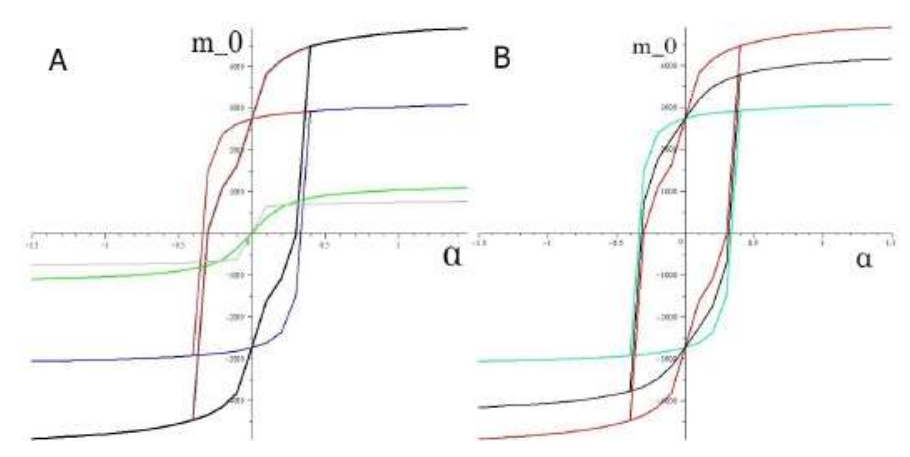

Figure 22. $R=3[\mathrm{~nm}]$. Nickel. $T=400[\mathrm{~K}]$. (A) - Three-layer ferromagnetic patterns with showing the way of each curve changes. Black and brown curves - total nanoparticle magnetization. Red and blue bulk layer magnetization. Light-green - surface layer. Silver - intermediate layer. (B) - Single-layer, two-layer and three-layer ferromagnetic patterns comparison. Red - three-layer total magnetization. Black - two-layer total magnetization.

methods can be adapted for any functions transformations, not for a narrow range of computational tasks.

\section{Conclusion}

We develop the Heisenberg theory and propose its applications. Such a context for the applied and computational part of the theory and models motivates us to reproduce the main results and additional ingredients of the theory, such as the thermodynamic and statistical physics alternatives. We take the basic ferromagnetic metals for the illustration to be close to the known results. The given examples, however, show very interesting features of the hysteresis phenomena.

Basing on obtained results, we consider the following algorithm of magnetization patterns creation by the Heisenberg ferromagnetism theory itself and a perspective for further studies and usage: magnetization curves and hysteresis loops are successfully produced from transcendental solutions; it can be currently used for spherical nanoparticles magnetization modeling and, not less important, it can replace approximate or manual theoretical magnetization creations. Note again, that the resulting transcendental equations that are used in the magnetization curves computations are obtained at very large values of spin, hence the whole problem may be considered at quasiclassical approximation as it does in transition to continuous description. All the tasks are fully completed: firstly, a technique of theoretical hysteresis loops creation by the Heisenberg theory has been developed from solutions of systems of transcendental equations; secondly, an algorithm of theoretical magnetization curves and hysteresis loops creation for multi-layer spherical nanoparticles by the Heisenberg theory has been built; thirdly, a procedure of the analysis and corrections of theoretical hysteresis loops and magnetization curves has been constructed. They all gave a background of overall algorithm joining and structuring see also Appendix for the details. Then, the analysis of obtained results is formulated.

Namely, the magnetization upper Curie temperature is described by a concrete approximation, on the ground of which the estimation is done: the magnetization corresponds to the common values of material melting and fluently descends (Figure 2), converges with the curve without approximation by the same theory.

The model of multi-layered nanoparticle magnetization is developed and the resulting patterns are analyzed. Generally, the patterns accuracy depends on the magnitudes of parameters - for processing simplifications several techniques are used. The technique of mean magnetization per electron is separated in a corresponding section and the algorithmic part in Appendix. The mean magnetization per electron, multiplied on number of electrons, give similar patterns as for a nanoparticle bulk part - it simplifies processing and gives more accurate loops (Figure 20). Hysteresis loops of mean magnetization per electron or bulk nanoparticle layer correspond to empirical hysteresis loops for nanorods by points densities and curvature shapes. For enlarging of loops application diapason (for 


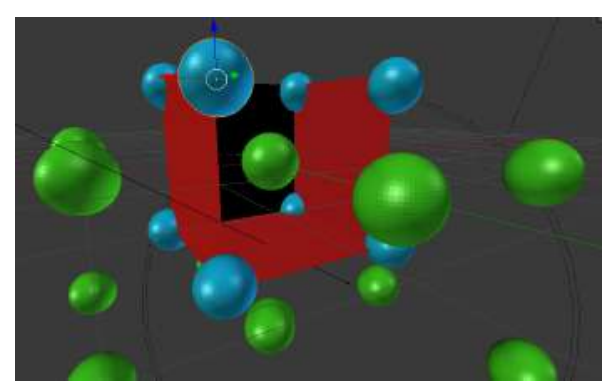

Figure 23. Defining the number of closest neighbors $z$ for Iron from its crystal structure. Bright-blue nodes, joined with red crystallographic planes, - cubic crystal system. Green nodes - volume-centered, located in a center of each cube.

wider range of materials) loop deformation coefficients could be created and applied. The mean magnetization per electron gave the next patterns on the example of Iron, Cobalt and Nickel (Figure 33-App[endix) - Iron element can be described by Heisenberg model as well; though, its pattern shows peculiar behavior in the hysteresis beginning range. It could be further fixed. The multi-layer magnetization model for a nanoparticle concretely demonstrates the impact of surface layers in total magnetization (results for paramagnetic patterns - Figure 15, Figure 16 and Figure 17, results for ferromagnetic patterns - Figure 21 and Figure 22). The most relevant nanoparticle radius for layer separation is approximately in a range of 3-5 nanometers, where the surface impact is significant, but does not exceed the bulk part.

Additionally, out of high processing magnitudes algorithm sometimes produced imprecise patterns areas - the solution is developed and presented too (Figure 43, Appendix). It brought us to creation of compound patterns analysis and correction stages, called subtraction and summation checking. This procedure and its principles are useful not only in particular patterns correction, but in transformations of primary correct patterns, such as curves smoothing, loops divisions etc. It opens a prospective of new coefficients defining, for other concrete patterns regions deformations. Such coefficients can account domain-wall structure, materials handling specificity etc. Exchange interactions, expressed by the exchange integral $J$ play a prominent role in ferromagnetic patterns creation, as well as the number of closest neighbors $z$ for each node. The dependence on temperature and material characteristics is concrete and accurate by Heisenberg theory. The technique of loop maximum/minimum curves separation is convenient and easy-to-use for any other functions transformations.

The overall algorithmic sequence allows applications in general field of hysteresis loops construction and analysis. Namely, the issue of hysteresis occurrence is solved numerically, and, not less important, the prolapse and peaks generated by discrepancies are controlled and eliminated. The example we trace is the hysteresis loops problem, appears in many areas of physical knowledge, so the algorithm can be useful in fragmentary reconstructions of other functions. The online available variant illustrates several sequences of common particular cases of loops and curves reconstructions [27].

\section{Appendix. Problems of computation: methods and details of solution.}

\subsection{On number of closest neighbors definition}

Here we put illustrations of the schematic atom positions visualizing, see Figs.23,24.

\subsection{Computation peculiarities and automatizing}

The peculiar step of computation automatizing - producing arrays by Maplesoft worksheet mode, as the manual creating of each curve has to be repeated 40 or, even 50 times for each section. First, 


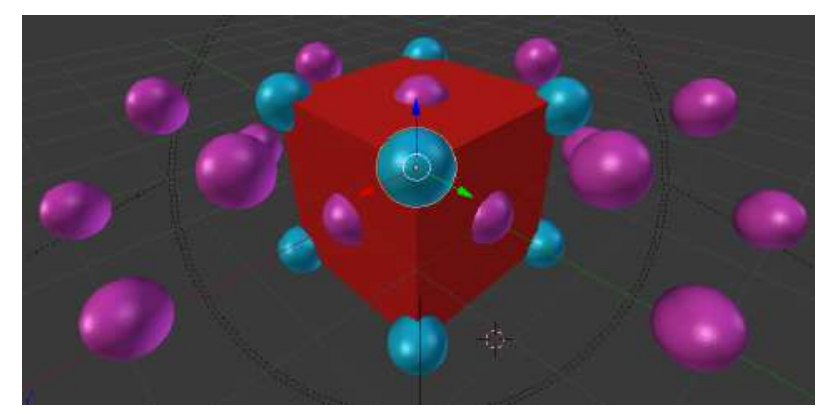

Figure 24. Defining the number of closest neighbors $z$ for Nickel and Cobalt from their crystal structure. Bright-blue nodes, joined with red crystallographic planes, - cubic crystal structure. Violet nodes face-centered, located in a center of each face.

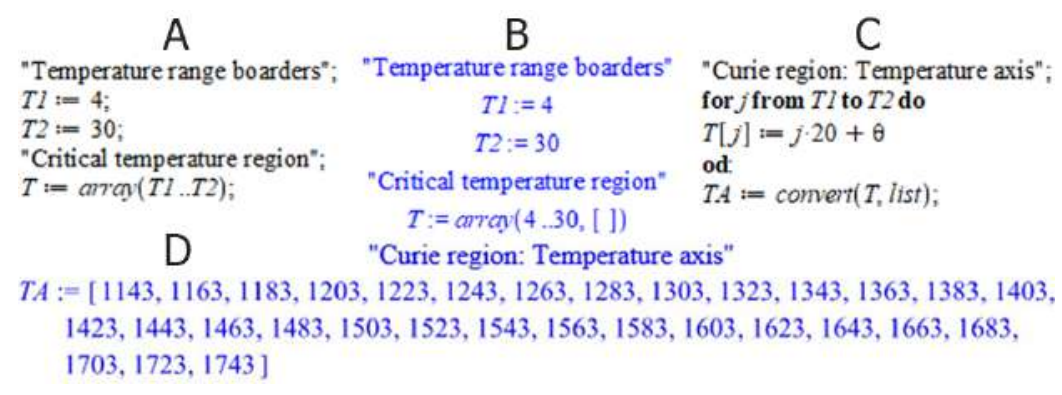

Figure 25. A temperature array creation by Maplesoft worksheet mode. (A) - The array creation command input. (B) - The array creation software reply. (C) - Array values evaluation by a sequential loop operator and recording them into the list. (D) - Array values evaluation software reply. The numbers for $T 1$ and $T 2$ denote the indexes of each future value. $T A$ - the resultant list of temperature changing values.

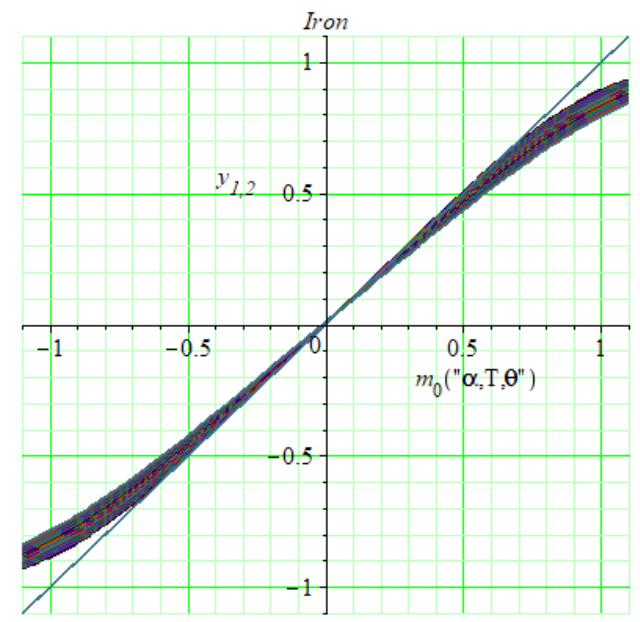

Figure 26. Graphical solution of the Heisenberg model without approximation with changing temperature over the Curie region (32). $T=1123 . .1723[K], \alpha=0.01, n=1$. The set of curves intersects the line function in descending of $m_{0}$ values. See Fig. 27 for a close-up view.

we have to create the changing temperature range. It is the simplest array among represented in this article, so let us start from its example, see Figure 25. The Figure 25 highlights two main stages of temperature array creation. The first stage is the creation of a set of values (A) and (B), which are to be evaluated in (C). Firstly, we establish two independent points, then the empty segment between them is fulfilled with ordered succession of integers. Index numbers for $T 1$ are specially selected in a way, transforming of which in $T[j]:=j \cdot 20+\theta$, give us the needed values. In such a case the first suitable number for us was $j=4$; it gave the first value of $T A$ list: $T A[1]:=1143$; (D). Coming back 


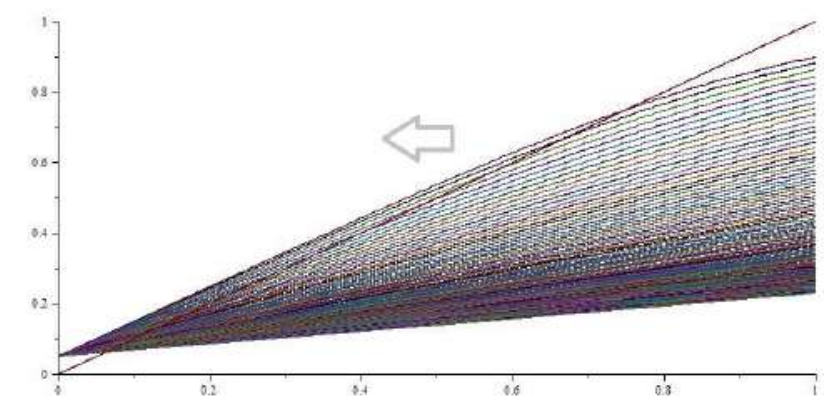

Figure 27. Graphical solution of Heisenberg model without approximation - Close-up view. The intersecting descends in the arrow direction with temperature increase. Functions tend to infinity at Curie point. the axis are the same as at Fig. 26.

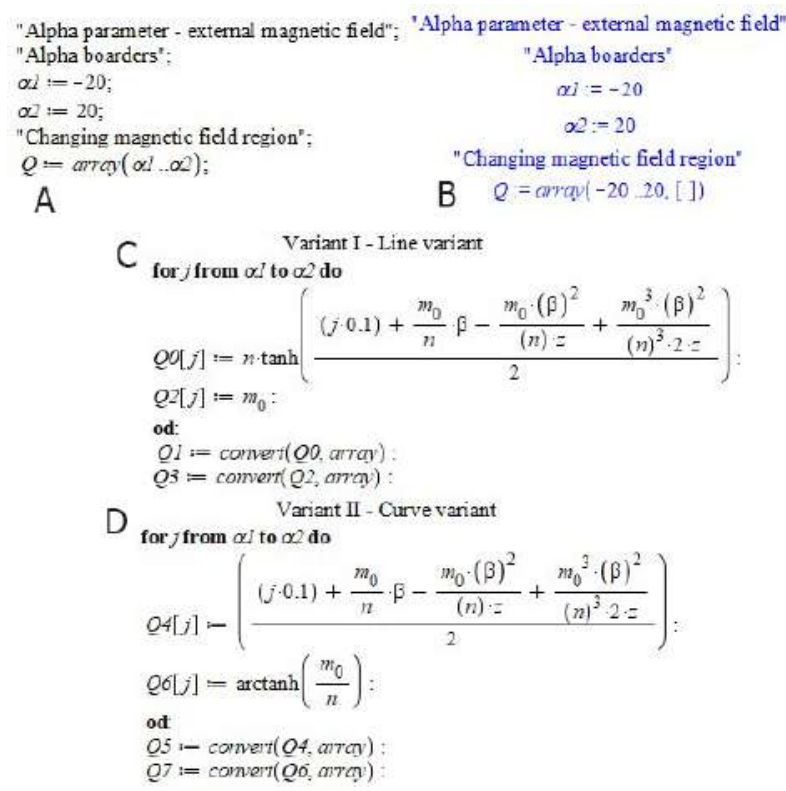

Figure 28. $m_{0}(\alpha)$ functions arrays and sets creation. (A) - Array creation command input. (B) - Array creation software reply. (C) - Functions set evaluation by the sequential loop operator. Variant I - (35). Variant II - (36). The numbers for $\alpha 1$ and $\alpha 2$ denote the integer indexes of each future value. $Q 1, Q 3$, $Q 5, Q 7$ - the resultant sets of four corresponding functions. $Q 3$ and $Q 7$ represent functions, which do not depend on changing $\alpha$ and $T$.

to intersection points lists creation, we produce the next loop operations, which are similar to the programming loops, created in Figure 28.

Then, after establishing of the borders, the interval between them is fulfilled one by one by other sequential indexes. Finally, the sequential loop operator do/od (C) transforms each $j$ index into the needed value of $\alpha$ by $\alpha \cdot 0.1$ operation. The figure 28 has its peculiarity in a compound loop evaluation. This time it implies the transformation of a single array into two arrays, the results are recorded in two different arrays instead of lists, as the list data type implies one solution for each index, while array data type can imply a set of solutions for each index. Composing the second array with so called "independent" functions (independent with respect to $\alpha$ and $T$ ) is the additional operation, which defines the individual intersecting function for every term with $\xi_{1}$.

Figure 29 (A) shows the possibility of materials combinations in Heisenberg model. Figure 29 (B) shows supplementary information about intersecting functions by $\beta$ parameter amplifications. 


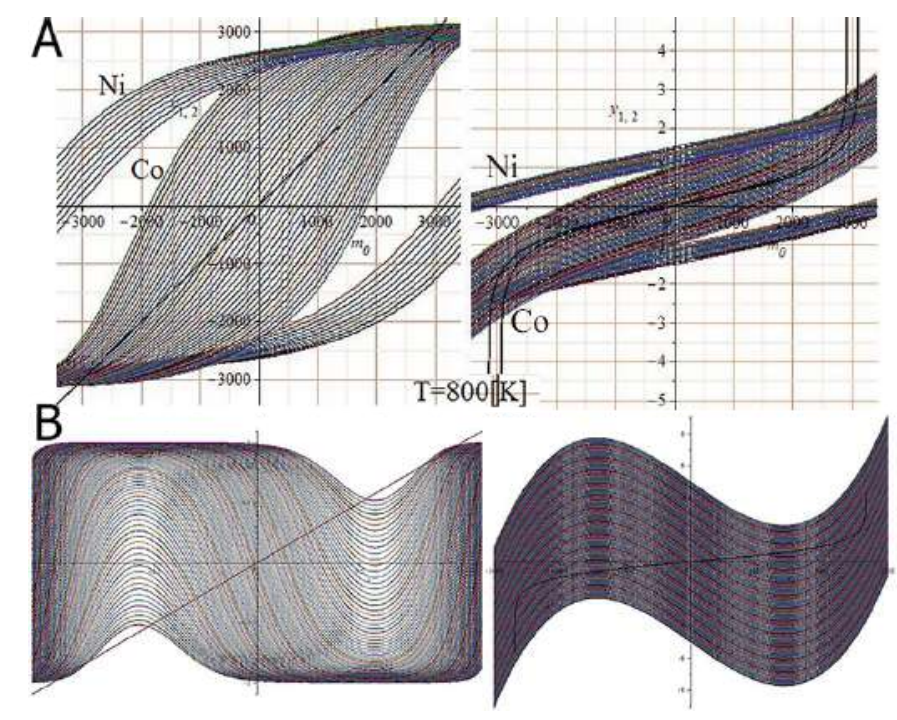

Figure 29. (A) - Heisenberg model combination prospective. The model could be used in irregularities mapping. The following combination is done with two materials - Nickel and Cobalt. Border $\alpha$ values are evaluated for Nickel, central - for Cobalt. (B) - Functions set mathematical behavior. Curve-to-curve and line-to-curve variants; $\alpha$ is taken in 0.001 increments for an arbitrary material; $\beta$ is amplified to 10 for defining the math reasons of a computational hysteresis origin.

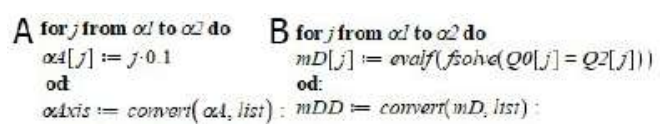

Figure 30. Programming loops implication. (A) - $\alpha$ axis creation. (B) - Numeric solutions evaluation.

\subsubsection{Heisenberg model combination prospective}

Each index of $Q$ primary array is transformed for evaluating $\alpha$ changing magnetic field imitation. The same is done with $m_{0}$ - main magnetic variable (in computations it is assigned as $m D$, emphasizing, that we work on drafts creation first) - Figure 30. During the $m D$ evaluation, computation records each function intersection one by one. It is illustrated in Figure 31. It brings visual prediction if there are no multiple solutions. For the manual example the following array $Q=\operatorname{array}(-25 . .25,[i])$ is chosen. The last shows, that there are 51 indexes in a primary array. For convenience, the list of maximum values is primary marked as a red curve, the list of minimum values is marked as a blue curve. The examples of loop drafts are displayed similarly as in Figure 4. The following graphs are figuratively called drafts, as they cover secondary processing and checks, if there occur any mistakes, peaks or prolapses. The secondary processing and checking are described in Subtraction checking section below (Section 6.3 ). Expressions from (38)-(40) do not need opening the brackets if they are evaluated via software. To calculate the number of electrons $\mathrm{n}$ by the means of Maple software package, we imply the next commands - Figure 34.

Float (round $($ Float $(x)))$ commands are put to use in discarding non-integer parts of a number, as every $n$ denotes a concrete set of elements nodes, which has to be integer in any cases. eval $f(x)$ command is primary intersected in the previous command, forming a compound request.

The loop occurrence checking repeats Figure 32 procedure, which founds out multiple intersections. If there are none, it displays the message $(C)$. This checking insures if the processing is done properly from the physical prospective - Figure 37. In case of incorrect operations or previous values re-writings it implements the second condition elif and warns users to restart operation. Such conditional loops if / else and if / elif are widely used in subtraction checking section for patterns secondary processing - Section 6.3. 


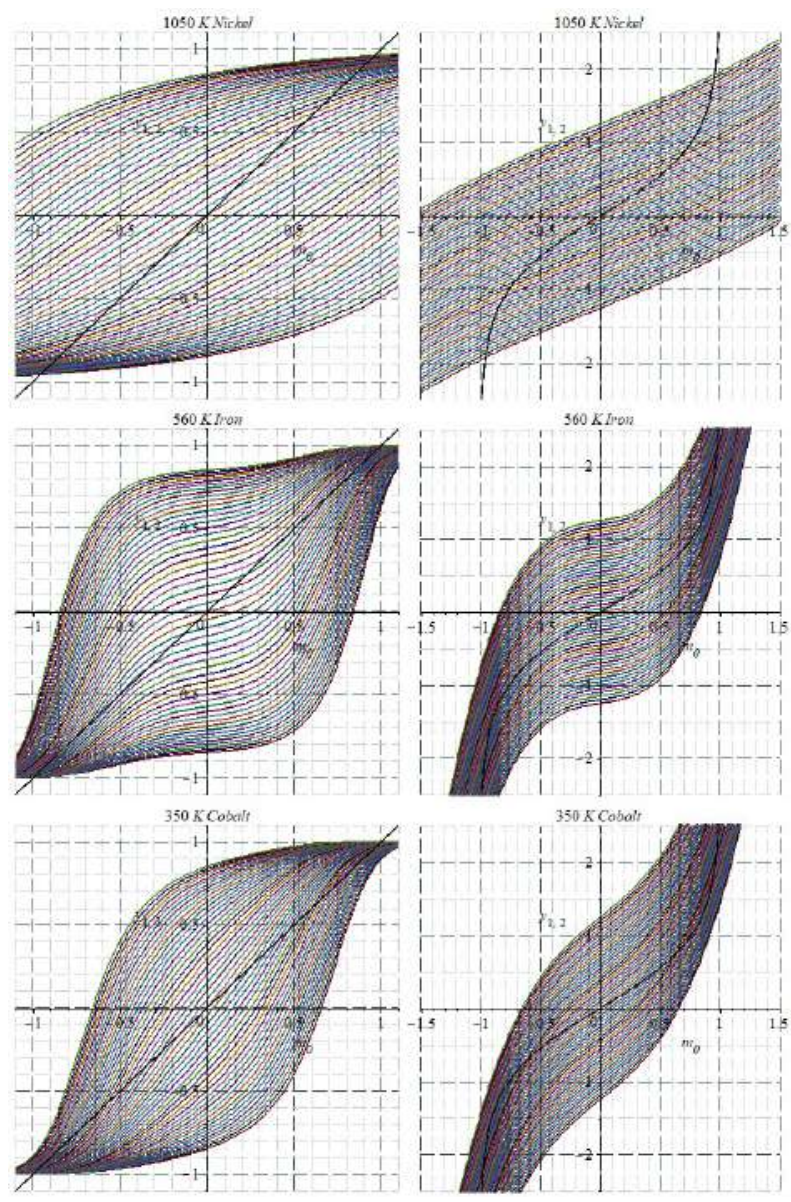

Figure 31. Solution process display. Left solutions - by (35) system of equations. Right solutions - by (36) system of equations. The same operation is done numerically in Figure 30 (B). The first pair shows the single solution for each curve. The second part shows the non-standard case for Iron (analogically with Figure 4). The third pair shows the homogeneous hysteresis, which gives rectangular loops. All the resulting patterns are illustrated here - Figure 33

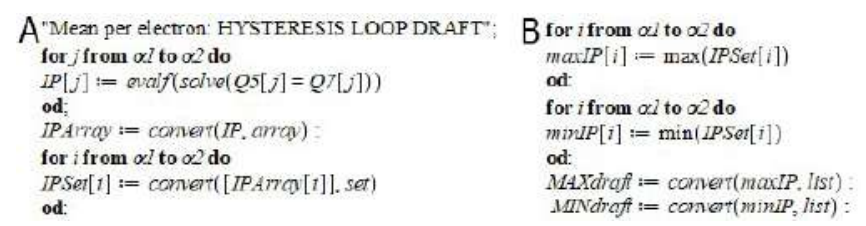

Figure 32. Maximum and minimum values separations. Four programming loops are involved. The first loop solves the system by command solve $(x)$ instead of $f$ solve $(x)$, which was previously used in producing ordinary curves. The result is rewritten in a new array. Each array line of values is transformed by the second programming loop into the set. As the result - functions set is created. Each set is processed by the third and the fourth loops, when among every set of values, it defines the biggest and the smallest value.

\subsection{On substruction}

The first subtraction stands in defining the module of subtractions of two closest points - Figure 39. This operation adverts to the magnetization curve pattern and works with its correction. As the subtraction is taken by module, all the peaks are presented in a positive part of a coordinate plane, relatively to the peak index axis. For now, we need an exact numeric, by which we will divide all the peaks into two groups. Low peaks are always proper; the highest peaks are always mistaken. This quality follows from the analysis of mistaken graphs - the drop between the closest intersection points 


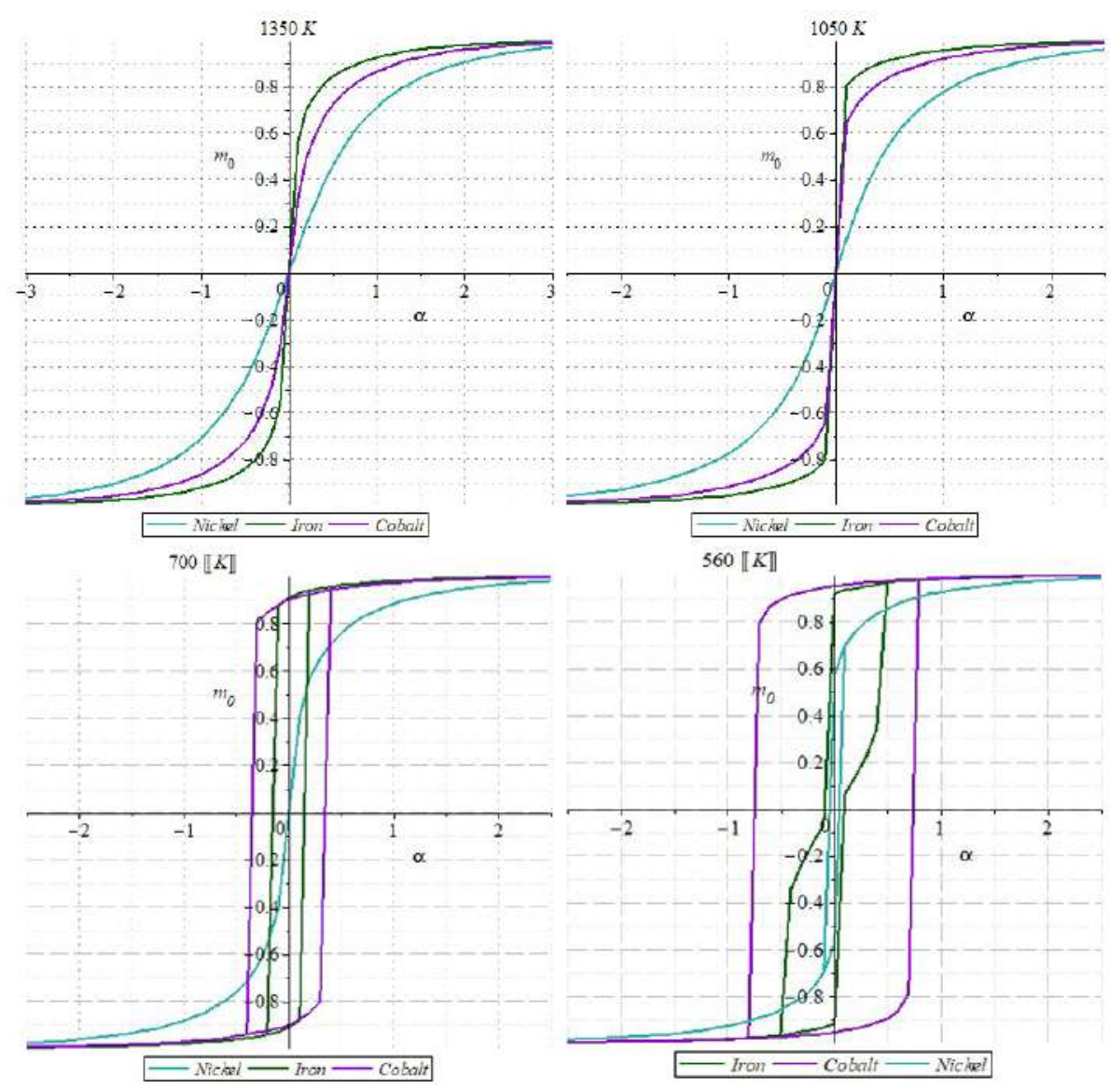

Figure 33. Magnetization patterns for Iron, Nickel and Cobalt in various temperatures. With the temperature decrease appears hysteresis. Each temperature range is written below the correspondent graph. As we see, Iron gives a fracture loop, as the functions set with $\xi_{1}$ is too curved to produce single-valued intersections.

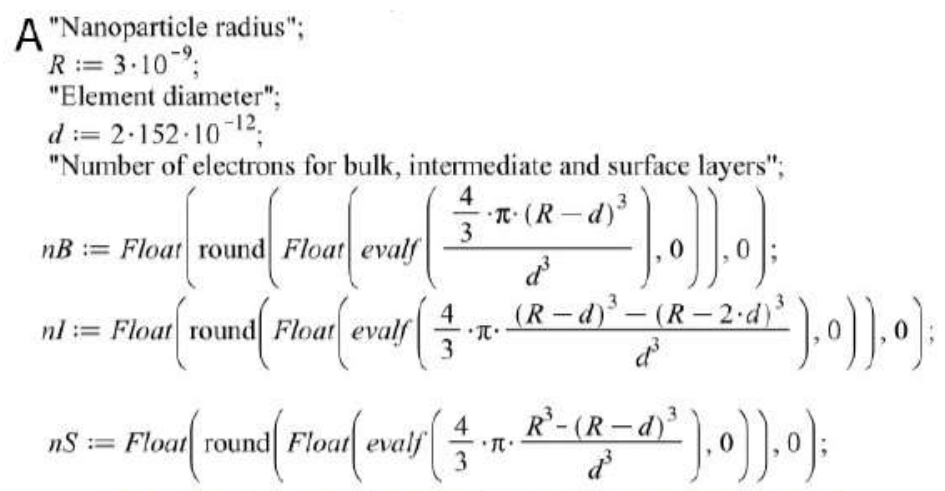

B "Number of electrons for bulk, intermediate and surface layers"

$$
\begin{aligned}
n B & :=2922 . \\
n I & :=881 . \\
n S & :=1104 .
\end{aligned}
$$

Figure 34. Number of electrons evaluation for each layer. $n_{B}$ - number of electrons for a bulk layer, $n_{I}$ - number of electrons for an intermediate layer, $n_{S}$ - number of electrons for a surface layer. A) Commands input order, B) Software reply. The dots after evaluated values denote the order of discarding non-integer parts of each number.

has to be organic, smooth, as in early presented curves - Figure 42. During the first subtraction we need only one numeric value to cut off the peaks over this numeric (marked by brown circle). This 


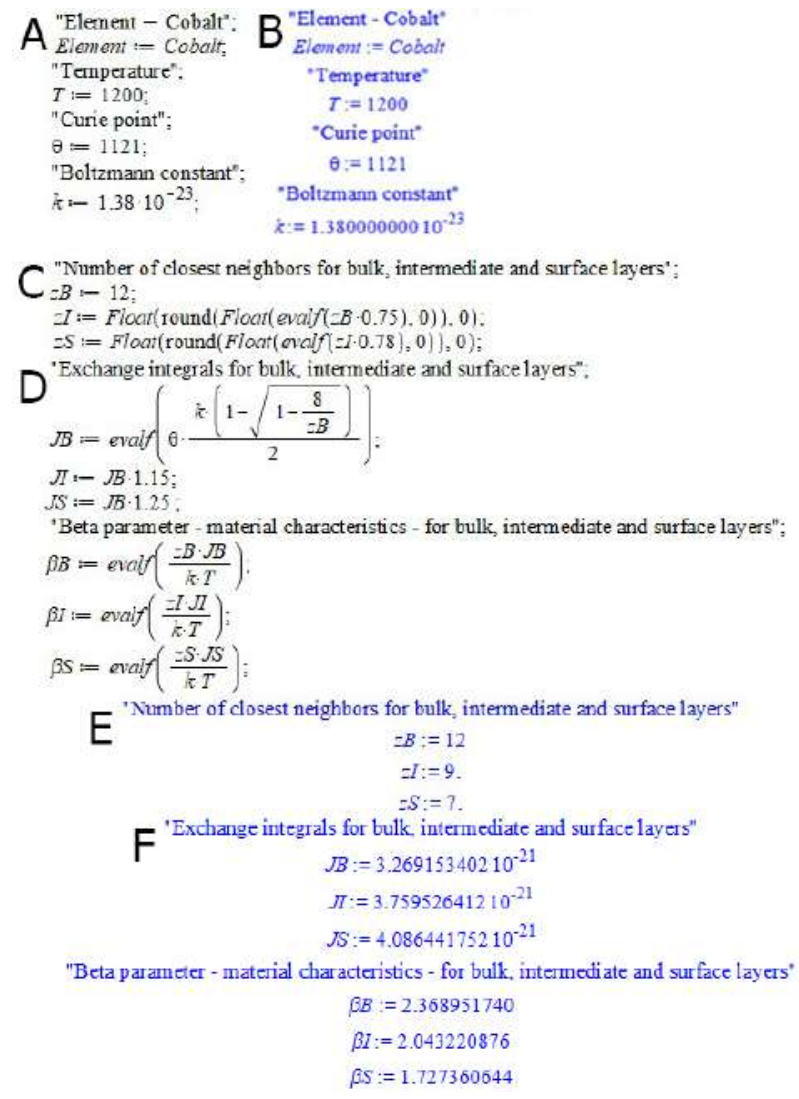

Figure 35. Material characteristics evaluation. (A), (C), (D) - command input. (B), (E), (F) - software reply and evaluation results. Resulting $\beta_{B}, \beta_{I}, \beta_{S}$ are of magnitude $1, J_{B}, J_{I}, J_{S}$ of order $10^{-21}$, which comports with the Heisenberg theory.

numeric coefficient is calculated through the median of all first subtractions results, multiplied on a percentage multiplier - Figure 40. As the prolapses and peaks originate in random, the percentage multiplier can fluctuate, depending on the particular case.

The loop is to be separated into five areas - Figure 43 (B). The first area contains two first maximums, again, for avoiding extra processing of non-existent values with indexes, smaller than the first index of each array $\alpha 1$. The fifth area contains two last minimums, for avoiding extra processing of non-existent values with indexes, bigger than the last index of each array $\alpha 2$. For example, if the correction algorithm is to be expanded for more complicated aims, the number of elements in a subtraction/sum can be bigger than two, so the number of points, separated in the first and the fifth areas, is to be the same. The first subtraction arrangement is clearly illustrated on the difference between two curve patterns for Nickel multi-layer model, where the surface pattern is correct and the intermediate pattern contains peaks - Figure 41 . Then, the division of a single curve could be done by the loop borders, if for the same situation both types of patterns are created - Figure 42 . MaxPeakList and MinPeakList are the assignations of hysteresis loop peak lists, which are created in the same manner as in the previous case.

In central part rebuilding for a single-curve pattern, the smoothness procedure is applied as well. It implies deriving new curve points by evaluation the mean value of several closest values of already corrected curve and several closest values of a correspondent auxiliary function, which is additionally assigned for each curve on the step of drafts creation - Figure 42.

Three central areas are left to be divided by some borders. Among these parts the subtraction modules of two closest maximums and two closest minimums are evaluated separately. On the base of each evaluation results, the mean numeric coefficients are derived for both curves. Each coefficient is multiplied on a percentage numeric multiplier, which can fluctuate on the assumption of random 


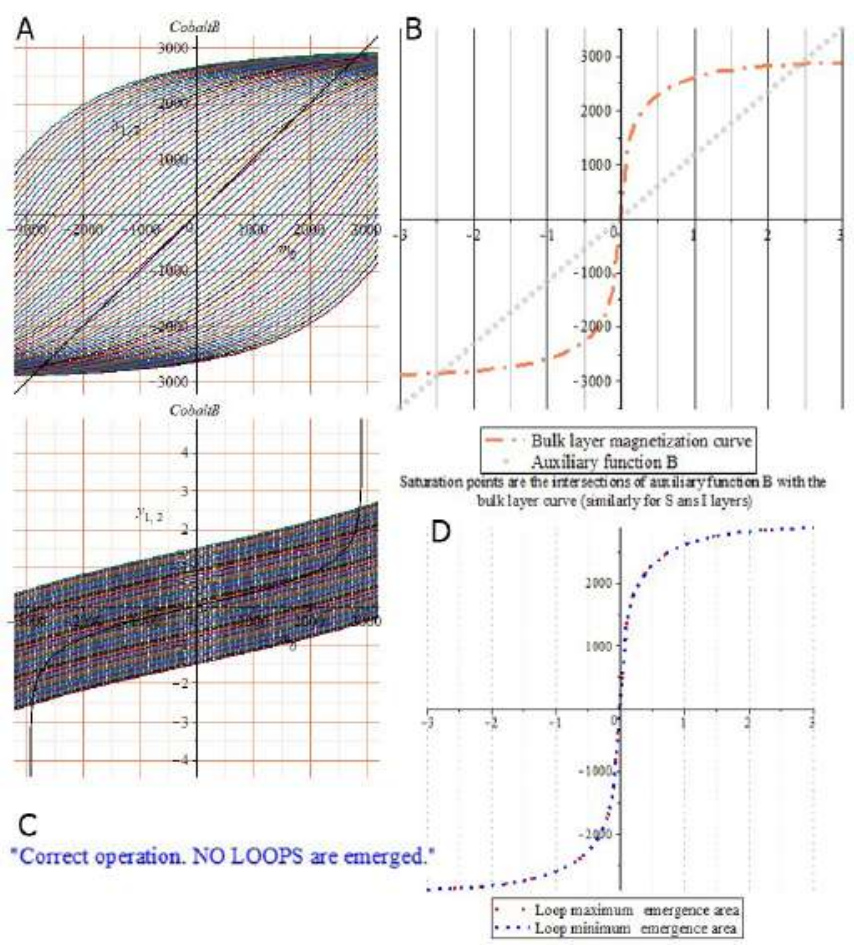

Figure 36. Paramagnetic bulk magnetization pattern creation. (A) - Intersection display. (B) - Processing result. (D) - Loop occurrence checking. (C) - Loop occurrence report display. Auxiliary function shows the saturation beginning by intersecting a magnetization pattern.

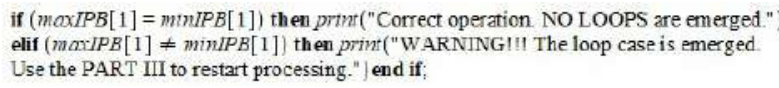

Figure 37. Programming loop with conditions if / elif.

"Intermediate layer. Peak axis creation";

for $i$ from $\alpha 1$ to $\alpha 2$ doIPeakNumb[i] := i od: IPeakAxis := convert(IPeakNuanb, list);

Figure 38. Defining the peak axis.

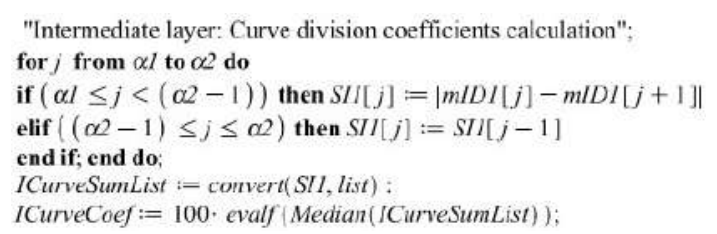

Figure 39. Subtraction module defining for each closest pair of points. $m I D$ is the analogue of $m D$ assignment of $m_{0}$ from previous sections, denoting a single-valued curve points values. Conditional loop operator if / elif used, it works in the same manner as if / else, but more than 1 condition can be implied with its usage.

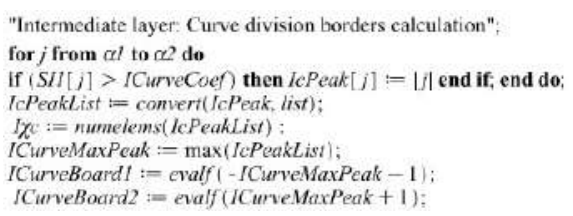

Figure 40. Mistaken peak list creation. The coefficient (marked as brown circle) from Figure 39 is used for values separation. The borders CurveBoard 1 and CurveBoard 2 "cut" the curves, for further separation of correct regions from mistaken regions.

mistakes occurrence - Figure 45. Every value of subtraction module is converted in a list, then this list is displayed with representing each module value and each module index. 


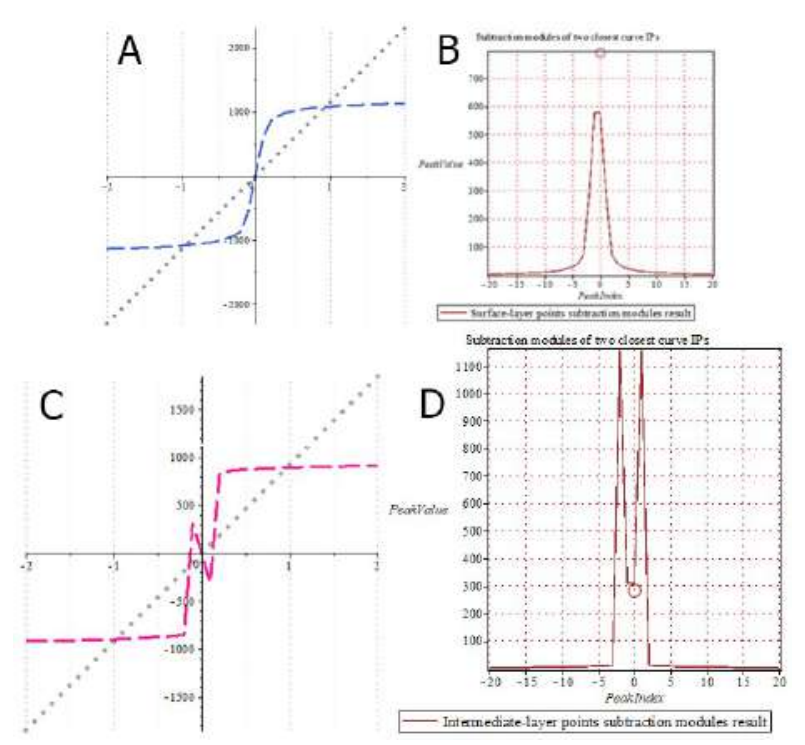

Figure 41. Curve peaks checking on the example of curves from Figure 19. As the curve coefficient (brown circle) is bigger than the highest peak, the curve is recognized as correct - (A) and (B). In case of mistakes, the coefficient cuts off the peaks over its value - (C) and (D). The results of reevaluation are presented in Figure 19, Figure 20 and Figure 21.

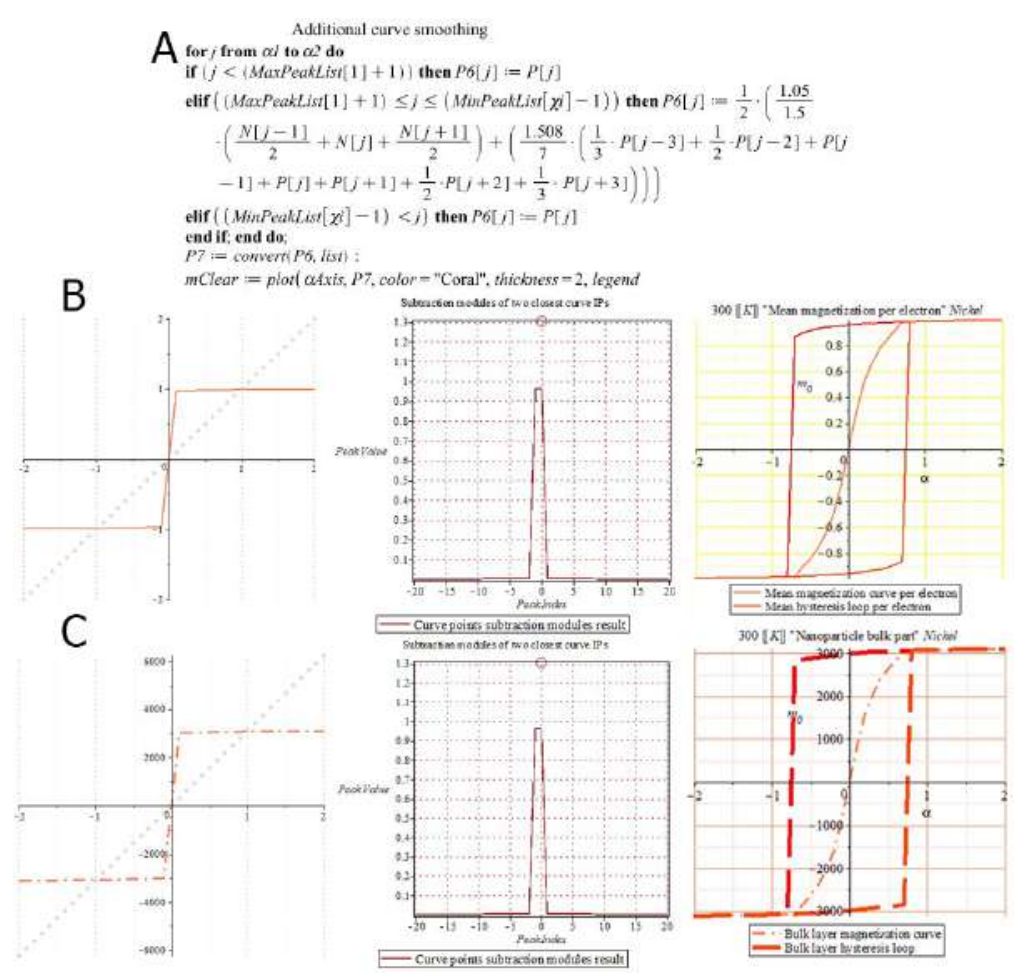

Figure 42. Curve smoothing by auxiliary functions (each point is marked as $N[j]$ ) and loop borders. As it is presented, the curves do not have the mistakes, but require smoothing. It is done with the help of subtraction checking. (A) - Curve parts separation could be done by hysteresis loop division borders too in case of simultaneous hysteresis and mono-curve occurrence, as it is presented here. (B) and (C): Here the auxiliary functions from previous sections are used for patterns smoothness calculation. It is done for mono-curves inside hysteresis loops, but could be used for loops smoothing as well, see also Sec. 6.4.

This situation is more complicated, comparatively to the single curve pattern, as the original loop always gives two correct peaks in areas of maximums/minimums transition from negative to positive 


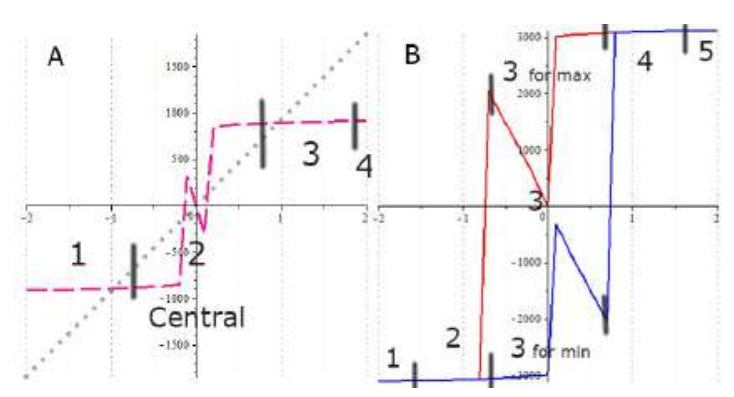

Figure 43. Curve and loop divisions. Subtraction checking works with the second region of a single curve (A) and with the third regions of each loop curves (B).

$$
\begin{aligned}
& \text { "Bulk layer: Additional loop reflection"; } \\
& P B S:=\operatorname{reflect}(B C l e a r M n,[0,0],[0,0]) \text { : }
\end{aligned}
$$

Figure 44. Loop reflection procedure. BClearMin assigns the curve of loop minimums in this case. This operation reflects the minimum values into maximums, or vice versa.

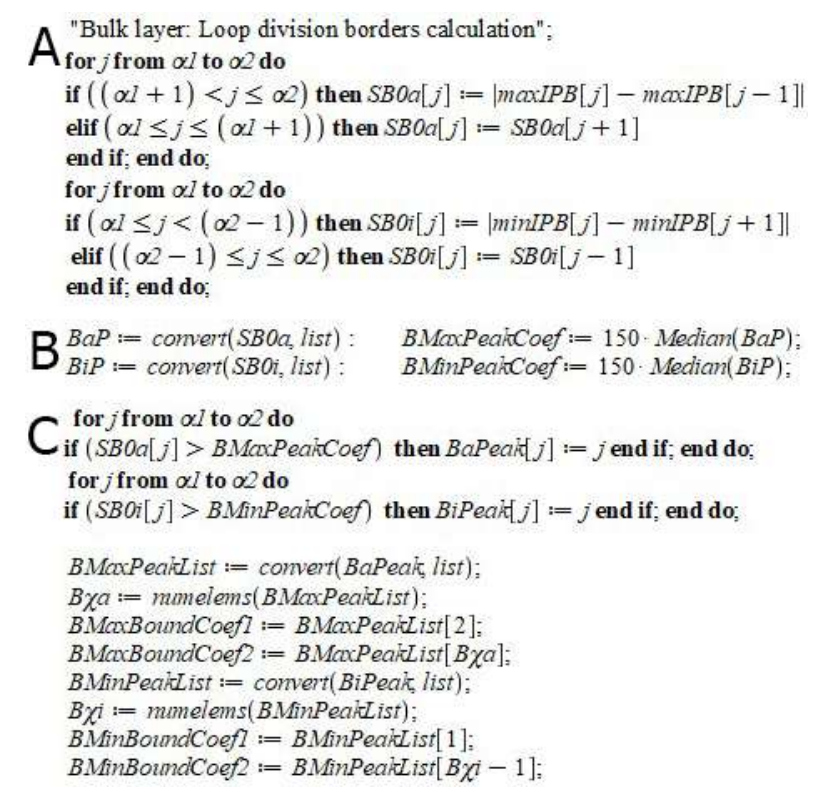

Figure 45. (A) - Second subtraction module defining. (B) - Peak coefficients calculation (marked by black circles). (C) - Peak lists creation for loop maximums and minimums.

(or vice versa). So, we create two corresponding lists of peak indexes and discard these two correct peaks from these lists. For maximums: the first peak is always correct, so we discard the first peak index from the list of loop maximum peaks. For minimums: the last peak is always correct, so we discard the last peak index from the list of loop minimum peaks. Among the region of all other peaks (locating in the center), their values are compared with a coefficient: when the coefficient is bigger than the peak value, it is not fixed as the border; when the coefficient is smaller than the peak value, it is fixed as the potential loop division border and is rewritten in a list of mistaken peaks. This procedure is illustrated here - Figure 46.

\subsection{The second subtraction}

The second subtraction could be used for loop division coefficients defining too, as in single-curve correction, but loop prolapse usually consists of multiple-pointed regions of mistakes, while a curve prolapse consists of one-two mistaken peaks. So, we use another method for loop coefficients evaluation - Figure 47. 


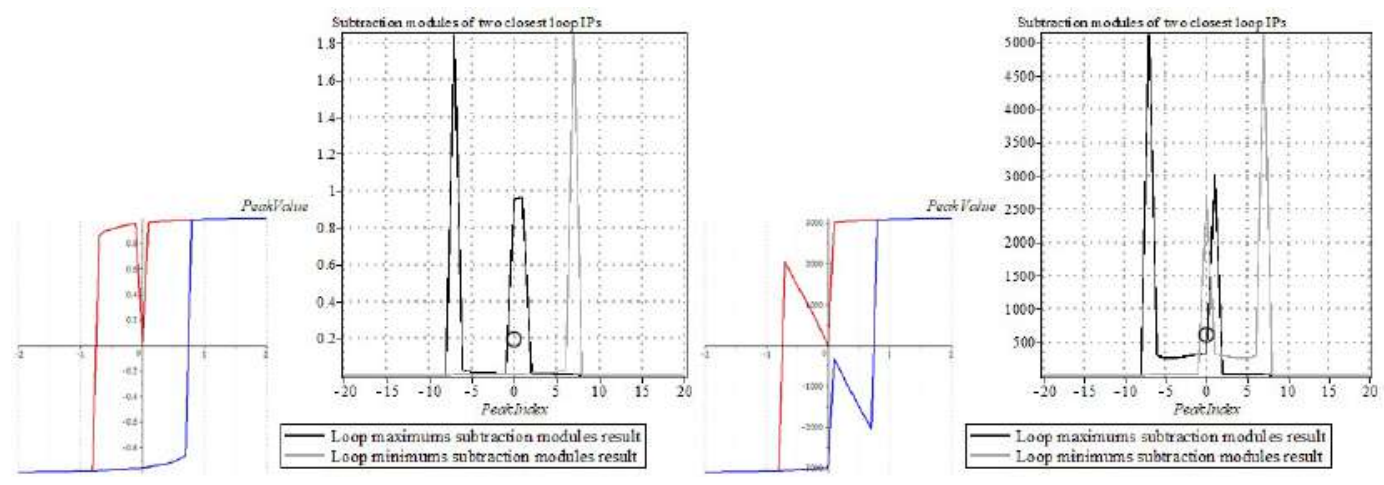

Figure 46. Loop borders defining by peaks. Black peaks show the maximums behavior (red), grey peaks show the minimums behavior. The first black peak and the last grey peak are correct, that is why they are not compared with the black point value (peak coefficient). Central peaks are discarded. The first sample is mean per electron model loop, the second - bulk nanoparticle loop, correction results of both are presented in Figure 42.

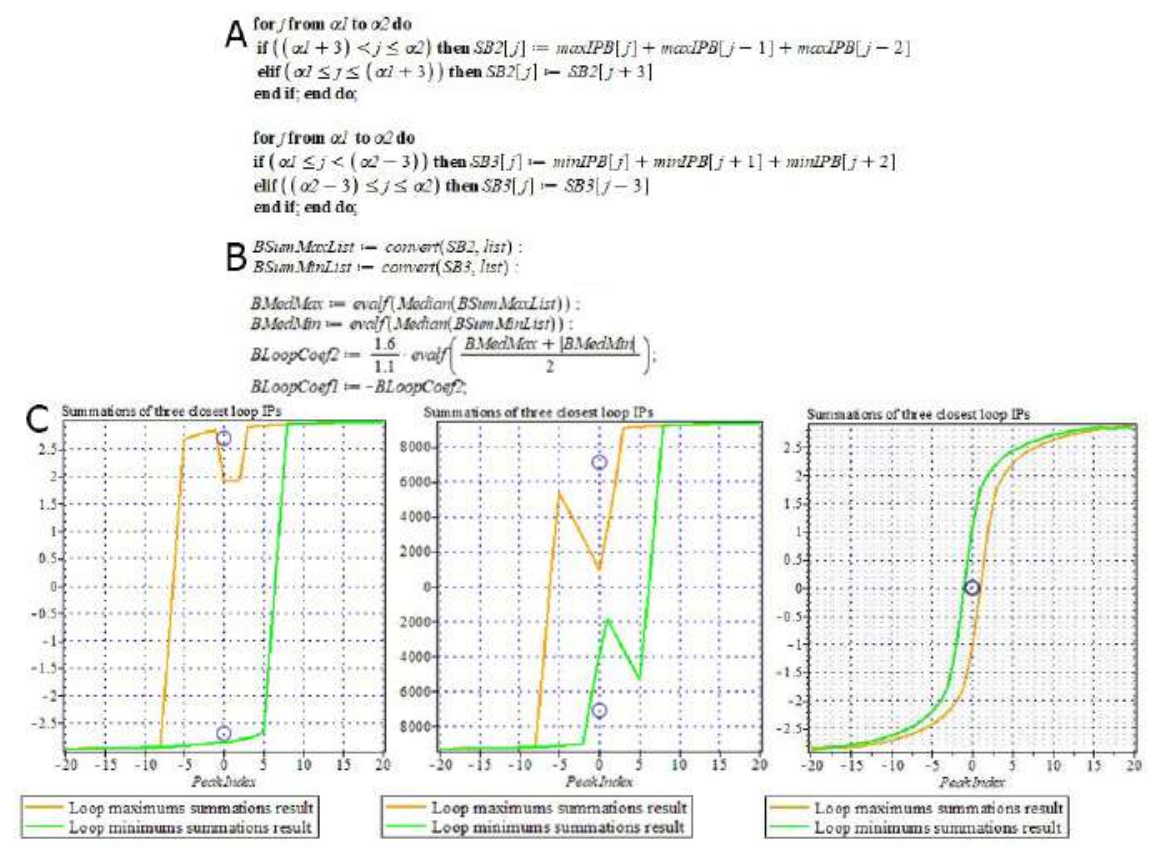

Figure 47. Summation checking. (A) - Summation code sample. (B) - Loop division coefficients evaluation. (C) - Software reply. First is for mean per electron loop correction, second is for bulk nanoparticle loop correction. Blue circles are loop division coefficients. The prolapse, which module is smaller than these coefficients, is discarded. In (C) different percentage multipliers are implied in a loop division coefficient evaluation, for illustrating the flexibility of subtraction checking procedure. The last of $(\mathrm{C})$ is the summation for a non-mistaken graph; it shows the prospective of hysteresis deformation; so the summation checking can be used not only for corrections, but as a deformation procedure of primary correct curves.

When we defined borders of loop division (four border indexes in total), we obtained, at which indexes maximum and minimum patterns will be subdivided (into three parts each pattern). For loop division coefficients evaluation, we create the sums of three closest values lists for both curves. Here the modules are not relevant, so the sums are just calculated. Three elements in the summation obligate us to cut off the first three index values from maximum sum list, and to cut off the last three index values from minimum sum list. For central symmetry saving we evaluate the medians of each list, then we find the mean value of two medians. This numeric is complemented by percentage multiplier, and it is duplicated with minus sign for negative loop values - it is done for saving symmetry too. 
As it was mentioned before, prolapses and peaks originate randomly, that is why in some cases the summations require recheck. For example, when there is only one mistaken peak for the whole loop pattern - all the borders match at one value then. The lacking borders can be defined manually, but there exists one more computational solution. All the obtained barders are repeatedly processed. If all the borders are already defined, algorithm displays the message on a screen, reporting: which concrete prolapse part of the whole magnetization pattern is properly processed. If the software lacks at least one index - it is immediately created at this step. Each border corresponds to one of two $\alpha$ indexes region. If the border has to be in negative $\alpha$ part, the reevaluation is done in negative $\alpha$ region only and vice versa. For single curve borders, it finds out the mean of three curve values in corresponding $\alpha$ region. The index of each value can be changed, if we want to change the curvature of a pattern by applying the other mean value. Then it finds out between which closest values this mean value is located. For loop division borders there are two reevaluation situations. If the computing lacks at least one coefficient, simply, it finds out the mean among the loop values in corresponding $\alpha$ region. Then, it computes, between which closest values this mean is located. If the loop borders match - representing the same index, both of indexes are changed into relevant correct loop peaks (which were previously discarded). It means, that each loop curve is divided then into three parts by its correct peaks - the points, where magnetization pattern changes the sign.

When all the coefficients and borders are obtained, the whole magnetization pattern is cut by the border indexes and the problematic parts are replaced by recently evaluated alternatives. Each pattern substitution is reevaluated individually by implying original manual formula. At low magnitudes of patterns, the loop reevaluation formulas could contain the mean of 5-6 elements, which produces the smoothing. The percentage multiplier from each reevaluation expression could be changed manually, in case of new pattern deviation. Though, the programming loop operators contain the replacement of values, which are not evaluated yet. It is counted on the repeated usage of this step. For the first time of applying - one amount of points is calculated, for the second time of applying the same step lacking amount is calculated, using the results of previous evaluation. The whole algorithm is built in a manner, where additional repeating of particular steps does not confuse or break down general modeling.

\subsection{Fragmentary reconstruction. Subtraction and summation checking}

\subsubsection{Prolapse origin reasoning}

As the graphs by (60) and similar transcendental systems of equations are not an ordinary task for software packages, in case of hysteresis, plots are sometimes displayed with prolapses. The same situation can happen in mono-curve cases too (corresponding to a paramagnetic magnetization pattern).

This defect occurs with loops as a consequence of nonstandard computation task: during the solution of systems, up to 5 intersections for each pair of functions are usually found out. The typical task for common transcendental solutions usually implicates less number of intersection points (ex.: 1 or 2 per curve), easier functions (ex.: trigonometric), or, even, involves the processing of much less amount of functions (usually 2-3 functions are processed simultaneously) than the represented variant in this work [37]. The current task imitates a detailed change of the magnetic field force, that leads to intersecting of two arrays of functions, one of which differ in tiny increments. That is why the algorithm omits some points, without accounting them in lists. As the result, the plots can be displayed with peaks or prolapses. The other defect, dealing with ordinary single-pointed curves, originates if the computation scale is very large. It is hard to process data with huge magnitudes $\left(10^{29}\right.$ order, for example), especially if the hardware is not primary purposed for this aim.

It is easy to prove, that the observed peaks and prolapses are built mistakenly. The first evidence is - exists the full observable central symmetry of all possible solutions. When the mistaken omission happens - the symmetry is contravened. On this ground all the intersections points recording is always 
done in exclusively one sequence - from $\alpha 1$ to $\alpha 2$. The second evidence is - visual comparison of an automatic list of i-points and their manual analogue. Before the development of automatizing, all the points were written out by hand, so the first loops were built manually. The last is the reason, why the following modeling is recommended to be used in its simplest configurations with small magnitudes. It was found out in the result, the matching curves with various scales are identical, so the difference is always in their axes scale, not in a shape of graph increments.

It is important to mention, professional and narrowly targeted for transcendental tasks packages should solve the system without highlighted prolapses. If such difficulty still arises, the detailed succession how to correct the graphs is outlined here and some recommendations are included as well.

6.5.2. Fragmentary reconstruction in use. Subtraction and summation checking application in magnetization patterns

The following correction stage is considered to be the most complicated, as it implies complex smoothing of the sharpest peaks. Though, all prolapses fixing is recommended to start from here.

It is called the subtraction and summation check procedure, as several subtractions and summations are used throughout problem solution. It implies the division of the whole magnetization pattern into segments with the help of coefficients and border indexes. Each coefficient contains a percentage multiplier, which provides the possibility of its value easy manual changing. In case of checking step improvement, the coefficients can be definitely established on the base of multitude prolapsing patterns coefficients generalization.

Before data converting we are to create the peak axis. It numerates each peak by its index number. As it is a draft step of algorithm, we can leave the $\alpha$ axis by the way of subtraction/summation argument. The following alternative just makes visualization more precise - Figure 38.

When correct peaks indexes are rewritten in a new list, our task is to define the borders of curve cutting. Each function pattern is cut into several areas. Namely, into four areas, but the fourth area always contains only two last points of a list and it is created just for avoiding extra processing of not-existent values with indexes, higher than the last index of each array $\alpha 2-$ Figure 43 (A). The other three areas are separated by borders in order not to influence the regions after saturation (saturation is always precise in every region), during changing the central part. The processing catches the first and the last peaks from this list and nominates the next border role for two future borders: index before the first peak index and index after the last peak index. Though, both borders have to be centrally symmetrical for an ordinary single-pointed curve, so there are some additional operations for border reflections, described in the next paragraphs of this section. If the curve draft is primary correct, the subtraction checking step can be omitted or both the borders could be set equal to 0 at this step. It leads us to "cutting" the correct curve in its center, so the central correction region will contain 0 points.

The second subtraction and the third procedure (summation) are developed for corrections of hysteresis loops. They work analogically to the curve pattern correction. The main difference is in separate checking of loop maximums (marked red) and loop minimums (marked blue). The corrections can be done for one loop part only, and then it can be reflected, producing the same resulting loop Figure 44 .

However, the aim of this step of work was to develop this checking with its future prospective in hysteresis biases modeling as well. Or, even, to forecast such subtraction/summation applications in other functions building, without any limitations due to their symmetry.

\section{References}

1. Bertotti, G. Hysteresis in Magnetism. Academic press, San Diego, 1998, p. 379.

2. Cardelli, E. Handbook of Magnetic Materials, Chapter 4. Advances in Magnetic Hysteresis Modeling 2015, 24, 323-409.

3. Perelomova, A. Hysteresis curves for some periodic and aperiodic perturbations in gases. Can. J. Phys. 2014, 92, 1-6. 
4. Perelomova, A. Hysteresis curves and loops for harmonic and impulse perturbations in some non-equilibrium gases. Cent. Eur. J. Phys. 2014, 11, 1541-1547.

5. Lee, H.; Chung, C. Effect of Electron Energy Distribution on the Hysteresis of Plasma Discharge: Theory, Experiment and Modeling. Sci. Rep. 2015, 5, 15254.

6. Schmool, D.; Markó, D. Magnetism in Solids: Hysteresis. Elsevier Inc. Reference Module in Materials Science and Materials Engineering. Chapter 11413 2018, p. 25.

7. Stoner, E.; Wohlfarth, P. A mechanism of magnetic hysteresis in heterogeneous alloys. Phil. Trans. Roy. Soc. 1948, A240, 599-642.

8. Guimaraes, A.P. Principles of Nanomagnetism. Springer 2009.

9. $\quad$ Skomski, R. Nanomagnetics. J. Phys.: Condensed Matter 15 2003, R841, 896.

10. Reichl, L. A modern course in statistical physics. A Wiley-Interscience publication, John Wiley and Sons, inc. 1998, 2nd Edition, 822.

11. Heisenberg, W. Zur Theorie des Ferromagnetismus. Zs. Phys. 1928, 49, 619-636.

12. Heitler, W.; London, F. Wechselwirkung neutraler Atome und homöopolare Bindung nach der Quantenmechanik. Zs. Phys. 1927, 44, 455-472.

13. Yamanouchi, T. On Heisenberg's Theory of Ferromagnetism. Proceedings of the Physico-Mat. Society of Japan. 3rd Series 1937, 19, 1003.

14. Leble, S. Heisenberg chain equations in terms of Fockian covariance with electric field account and multiferroics in nanoscale. NANOSYSTEMS: PHYSICS, CHEMISTRY, MATHEMATICS 2019, 10, 13.

15. Heitler, W. Staorungsenergie und Austausch beim Mehrkaorperproblem. Zs. Phys. 1927, 46.

16. Heitler, W. Zur Gruppentheorie der homopolaren chemischen Bindung. Zs. Phys. 1928, 47, 835.

17. Nakano, H. The Heisenberg Theory of Ferromagnetism of the Crystal constituted by the Atoms with Spin One. Progress of Theoretical Physics 1953, 9, No.4.

18. Leble, S. Magnetoelectric effects theory by Heisenberg method based on permutation group symmetry of nanoparticles. NANOSYSTEMS: PHYSICS, CHEMISTRY, MATHEMATICS 2020, 11, 50-64.

19. Lakshmanan, M. The fascinating world of the Landau-Lifshitz-Gilbert equation: an overview. Phil. Trans. R. Soc. A 2011, 369, 1280-1300. doi:10.1098/rsta.2010.0319.

20. Landau, L.; Lifshitz, E. On the theory of the dispersion of magnetic permeability in ferromagnetic bodies. Phys. Z. Sowjetunion 1935, 8, 101-114.

21. Martinez-Garzia, J.; Rivas, M.; Garcia, J. Induced ferro-ferromagnetic exchange bias in nanocrystalline systems. Journal of Magnetism and Magnetic Materials 2014, 377, 424-429.

22. Martinez-Garca, J.; Rivas, M.; Garcia, J. Induced ferro-ferromagnetic exchange bias in nanocrystalline systems. Journal of Magnetism and Magnetic Materials 2015, 377, 424 - 429. doi:https://doi.org/10.1016/j.jmmm.2014.10.123.

23. Kolesnikova, V.; Martinez-Garca, J.; Rodionova, V.; Rivas, M. Study of bistable behaviour in interacting Fe-based microwires by first order reversal curves. Journal of Magnetism and Magnetic Materials 2020, 508, 166857. doi:https://doi.org/10.1016/j.jmmm.2020.166857.

24. Omelianchik, A.; Singh, G.; McDonagh, B.H.; Rodionova, V.; Fiorani, D.; Peddis, D.; Laureti, S. From $\mathrm{Mn3 \textrm {O }} / \mathrm{MnO}$ core-shell nanoparticles to hollow MnO: evolution of magnetic properties. Nanotechnology 2018, 29, 055703. doi:10.1088/1361-6528/aa9e59.

25. Gritsenko, C.; Dzhun, I.; Volochaev, M.; Gorshenkov, M.; Babaytsev, G.; Chechenin, N.; Sokolov, A.; Tretiakov, O.A.; Rodionova, V. Temperature-dependent magnetization reversal in exchange bias NiFe/IrMn/NiFe structures. Journal of Magnetism and Magnetic Materials 2019, 482, 370 - 375. doi:https://doi.org/10.1016/j.jmmm.2019.03.044.

26. Rungger, I.; Sanvito, S. Ab initio study of the magnetostructural properties of MnAs. Phys. Rev. B 2006, 74, 024429. doi:10.1103/PhysRevB.74.024429.

27. Chychkalo, N.; Leble, S. Fragmentary reconstruction of functions by functions values summations on the example of Iron, Nickel and Cobalt spherical nanoparticles by Heisenberg ferromagnetism theory. Notes to master thesis, unpublished 2020.

28. Bertotti, G.; Seprico, C. Nonlinear Magnetization Dynamics in Nanosystems. Elsevier Science 2008.

29. Heisenberg, W. Zur Theorie des Magnetpstriktin und der Magnetisierungkurve. Zs. Phys. 1931, 69, $287-297$.

30. Spaldin, N. Magnetic Materials: Fundamentals and Applications. (2nd ed.). Cambridge: Cambridge University Press 2010, p. ISBN 9780521886697. 
31. Brandão, J.and Dugato, D.; Seeger, R.; Denardin, J.; Mori, J. A.; Cezar, J.C. Observation of magnetic skyrmions in unpatterned symmetric multilayers at room temperature and zero magnetic field. Sci Rep 2019, 9, 4144. doi:10.1038/s41598-019-40705-4.

32. Baraban, I.; Leble, S.; Panina, L.; Rodionova, V. Control of magneto-static and -dynamic properties by stress tuning Fe-Si-B amorphous microwires with fixed dimensions. Journal of Magnetism and Magnetic Materials 2019, 477, 415-419.

33. Gandha, K.; Elkins, K.; Poudyal, N.; Liu, X.; Liu, J. High energy product developed from cobalt nanowires. Scientific reports 2014, 4, No 5345.

34. Vonsovskij, S. Magnetism, Magnetic properties of dia-, par-, ferro-, antiferro- and antiferrimagnetics. Moskow: Nauka 1971, p. 1025.

35. Frenkel, I.; Dorfman, J. Spontaneous and Induced Magnetisation in Ferromagnetic Bodies. Nature 1930, 126, 274-275.

36. Brown, W. Comparing community structure identification. Jr. Appl. Phys. 1940, 11, 160-172.

37. Zaguskin, V. Handbook of Numerical Methods of the Solution of Algebraic and Transcendental Equations. Oxford: Pergamon Press 1961, p. 193. 